\title{
JIP1 Mediates Anterograde Transport of Rab10 Cargos during Neuronal Polarization
}

\author{
Cai-Yun Deng, ${ }^{1}$ Wen-Liang Lei, ${ }^{1}$ Xiao-Hui Xu, ${ }^{1,2}$ Xiang-Chun Ju, ${ }^{1}$ Yang Liu, ${ }^{1}$ and Zhen-Ge Luo ${ }^{1}$ \\ ${ }^{1}$ Institute of Neuroscience and State Key Laboratory of Neuroscience, Shanghai Institutes for Biological Sciences, Chinese Academy of Sciences, Shanghai \\ 200031, China, and ${ }^{2}$ School of Life Sciences, Shanghai University, Shanghai 200444, China
}

\begin{abstract}
Axon development and elongation require strictly controlled new membrane addition. Previously, we have shown the involvement of Rab10 in directional membrane insertion of plasmalemmal precursor vesicles (PPVs) during neuronal polarization and axonal growth. However, the mechanism responsible for PPV transportation remains unclear. Here we show that c-Jun N-terminal kinase-interacting protein 1 (JIP1) interacts with GTP-locked active form of Rab10 and directly connects Rab10 to kinesin-1 light chain (KLC). The kinesin1/JIP1/Rab10 complex is required for anterograde transport of PPVs during axonal growth. Downregulation of JIP1 or KLC or disrupting the formation of this complex reduces anterograde transport of PPVs in developing axons and causes neuronal polarity defect. Furthermore, this complex plays an important role in neocortical neuronal polarization of rats in vivo. Thus, this study has demonstrated a mechanism underlying directional membrane trafficking involved in axon development.
\end{abstract}

Key words: Jip1; neuronal polarity; Rab10; vesicle trafficking

\section{Introduction}

Polarized membrane addition contributes to cell polarization in various cell types (Lecuit and Pilot, 2003). Neurons are highly polarized cells with a single long axon sending out information, and multiple and arborized dendrites receiving information. Usually, the length and area of the single axon are much larger than those of total dendrites, and thus it is believed that there is asymmetric delivery of membrane to these two subcellular compartments (Ye et al., 2006). In cultured hippocampal neurons, which have been used widely as a classical model to study axon specification and neuronal polarization, axon specification is preceded by asymmetrically faster growth of one neurite, which will later become the axon (Cáceres et al., 1986; Dotti et al., 1988). Interestingly, the post-Golgi vesicles are preferentially transported to this neurite before morphological polarization (Bradke and Dotti, 1997). Thus, membrane dynamics are not merely a passive aspect of neuronal morphogenesis, but rather play a positive role in triggering axonal-dendritic polarization.

It has been postulated that the post-Golgi pleiomorphic plasmalemmal precursor vesicles (PPVs) provide the sources for lipids and membrane proteins required for the polarized membrane supply (Pfenninger, 2009). Recently, we have shown that Rab10,

\footnotetext{
Received Oct. 22, 2013; revised Dec. 9, 2013; accepted Dec. 18, 2013

Author contributions: C.-Y.D. and Z.-G.L. designed research; C.-Y.D., W.-L.L., and X.-C.J. performed research; X.-H.X., X.-C.J., and Y.L. contributed unpublished reagents/analytic tools; C.-Y.D. and W.-L.L. analyzed data; C.-Y.D. and Z.-G.L. wrote the paper.

This work was supported by the National Natural Science Foundation of China (Grants 31330032, 31321091, and 61327902) and the Key State Research Program (Grant 2014CB910203). We thank Dr. Q. Hu of ION Imaging Facility for the microscope analysis.

The authors declare no competing financial interests.

Correspondence should be addressed to Dr. Zhen-Ge Luo, Institute of Neuroscience, Chinese Academy of Sciences, 320 Yue Yang Road, Shanghai 200031, China. E-mail: zgluo@ion.ac.cn.

DOI:10.1523/JNEUROSCI.4496-13.2014

Copyright $\odot 2014$ the authors $\quad 0270-6474 / 14 / 341710-14 \$ 15.00 / 0$
}

a member of the Rab family of small GTPases, is associated with PPVs (Wang et al., 2011), and that Myosin V interaction with Rab10 controls the sorting of PPVs from the trans-Golgi network (Liu et al., 2013). Accumulating evidence from cultured hippocampal neurons suggests that the directional and polarized membrane insertion occurs in the distal regions of developing axons (Futerman and Banker, 1996; Dupraz et al., 2009; Wang et al., 2011). However, how the Rab10-positive PPVs are delivered to the destination places is totally unknown.

Kinesin-1 is a conventional member of the kinesin family, which is composed of two heavy chains [kinesin heavy chain $5 \mathrm{~A}$ (KIF5A), KIF5B, or KIF5C] and two light chains [kinesin-1 light chain (KLC1) or KLC2; Cai et al., 2007; Verhey and Hammond, 2009]. It has been shown that kinesin-1 can associate with vesicles directly, or indirectly by interacting with various adaptor proteins, which determine selective transport of cargos in neurons (Setou et al., 2002; Kanai et al., 2004; Su et al., 2004; Glater et al., 2006; Blasius et al., 2007; Cho et al., 2007; Grigoriev et al., 2007; Taya et al., 2007; Arimura et al., 2009; Yamada et al., 2010; Huang et al., 2011). Among these adaptors, c-Jun N-terminal protein kinase (JNK)-interacting protein 1 (JIP1), a scaffold protein for JNK signaling pathways (Whitmarsh, 2006), mediates axonal transport of several cargos (Koushika, 2008). Interestingly, JIP1 is important for axonal development of cultured cortical neurons (Dajas-Balador et al., 2008). However, the mechanisms underlying JIP1 action in axon development and its role in vivo have not been explored.

Here, we show that JIP1 acts as a scaffold protein linking Rab10 to kinesin- 1 and mediates anterograde transport of Rab10-positive vesicles during axon development. This study has demonstrated a molecular mechanism for anterograde transport of Rab10-positive PPVs along microtubules to the destination sites, where membrane fusion occurs to control axon development. 
A

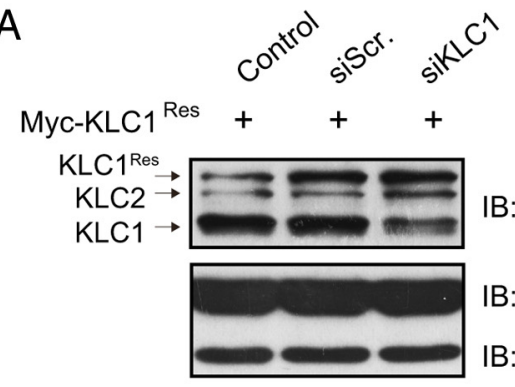

B

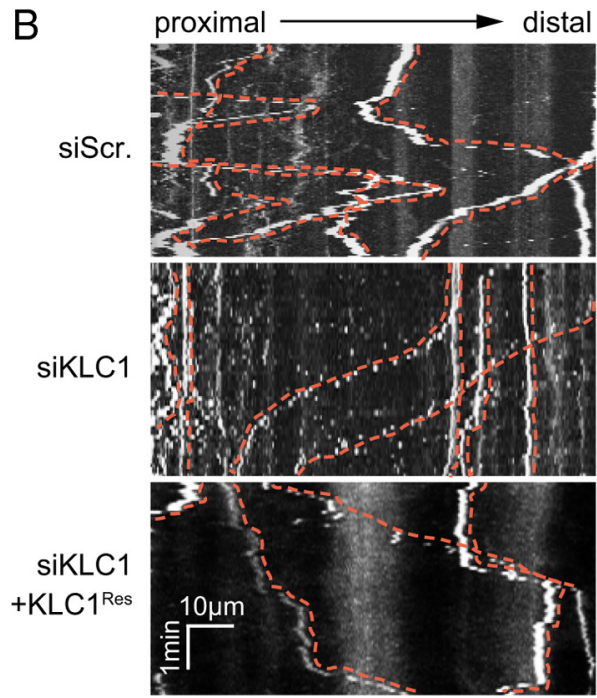

C

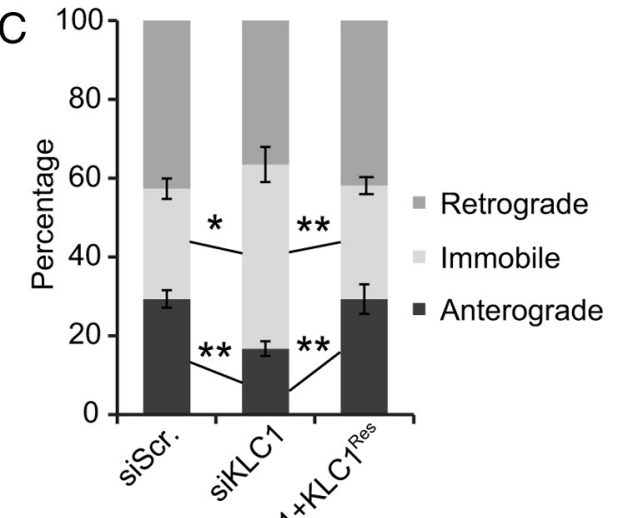

Figure 1. KLC1 is required for anterograde transport of Rab10-PPVs. A, Cortical neurons were transfected with pSUPER vector or pSUPER-encoding siKLC1 or siScr together with KLC1 Res . Endogenous KLC (KLC1 and KLC2) and KLC1 ${ }^{\text {Res }}$ levels were analyzed by IB with antibody against KLC. Actin and GAPDH were probed as controls. $\boldsymbol{B}$, Representative kymographs of TD-Rab10 vesicles in cultured hippocampal neurons transfected with siScr or siKLC1, either alone or together with KLC1 ${ }^{\text {Res }}$. Scale bar, $10 \mu \mathrm{m}$. C, Quantification for the percentage of anterograde, retrograde, or immobile TD-Rab10 vesicles in neurons transfected with siScr, siKLC1, or siKLC1 plus KLC Res. Data are presented as the mean \pm SEM. ${ }^{*} p<0.05,{ }^{* *} p<0.01$, Student's $t$ test. $D$, Quantification for the mean velocity of TD-Rab10 vesicles in axons of cultured hippocampal neurons transfected with indicated plasmids. Data are presented as the mean \pm SEM. ${ }^{*} p<0.05$, Student's $t$ test.

\section{Materials and Methods}

Reagents and antibodies. Poly-D-lysine (PDL) hydrobromide was from Sigma-Aldrich. Neurobasal and B27 media were from Invitrogen. Ni-NTA agarose was from Qiagen, protein A or protein G agarose was from Roche, and glutathione-Sepharose was from GE Healthcare Life Sciences. Antibodies used for immunostaining or immunoblotting (IB) were as follows: KLC (Millipore Bioscience Research Reagents), JIP1 (Santa Cruz Biotechnology), Rab10 (Proteintech Group and Abmart), GM130 (BD Biosciences), Smi312 (Covance), Ki67 (Abcam), Tbr2 (Abcam), HA (Sigma), Flag (Sigma), Myc (Santa Cruz Biotechnology and Millipore), glutathione $S$-transferase (GST; Proteintech Group), His (Abcam and Abmart), GFP (Invitrogen, Abmart, and Aves Labs), actin (Sigma), and GAPDH (KangChen), and DAPI (Beyotime Institute of Biotechnology). Alexa Fluor 488-, Alexa Fluor 555-, or Alexa Fluor 647-conjugated secondary antibodies against mouse or rabbit IgG [heavy and light chain $(\mathrm{H}+\mathrm{L})$ ] were purchased from Invitrogen. HRP-conjugated secondary antibodies against mouse or rabbit IgG $(\mathrm{H}+\mathrm{L})$ were from Millipore.

Constructs. Flag-JIP1 and HA-KLC1 constructs were provided by Dr. K. J. Verhey (University of Michigan Medical School, Ann Arbor, MI). JIP1 was subcloned into pCS2 + MT in frame with $6 \times$ Myc epitopes at the $\mathrm{N}$ terminus or into pGEX-2T to produce GST fusion proteins. KLC1 was amplified by PCR and subcloned into pECFP-C1 or pCS2+MT vectors, respectively. Different domains of KLC1 and JIP1 were inserted into pCS2 + MT with $6 \times$ Myc epitopes at the $\mathrm{N}$ terminus. The small interfering RNA (siRNA)-resistant form of KLC1 or JIP1 was generated using Site-Directed Mutagenesis Kit (Stratagene) without changing the amino acid sequences. TD-Rab10 was constructed by inserting tdTomato sequence into pKH3-Rab10 plasmid at the HindIII site. Constructs for other Rabs (Rab5a, Rab8a, Rab8b, Rab11, and Rab13) were introduced in the previous study (Wang et al., 2011).

Small interfering RNAs. siRNAs were designed against the rat KLC1 sequence $5^{\prime}$-ATACGACGACGACATCTCT- 3 ' and the JIP1 sequence $5^{\prime}$ GGTACCATCCAAAGAACAACA-3'. All double-stranded oligonucleotides against KLC1, JIP1, and scrambled sequences were synthesized by GenePharma (Shanghai, China), and were inserted between the BglII and HindIII sites of pSUPER vector.

Recombinant protein purification. Rab10 was subcloned into pET32a vector to produce His-tagged fusion proteins. JIP1 was subcloned into pGEX-2T vector to produce GST fusion proteins. Recombinant proteins were expressed in Escherichia coli BL21 (DE3) and purified using a NiNTA agarose column or glutathione-Sepharose, respectively.

Immunoprecipitation and pull-down assays. HEK 293 cells were cultured in DMEM supplemented with $10 \%$ fetal bovine serum and transfected with lipofectamine 2000 following the manufacturer's instruction. Transfected HEK293 cells were lysed in buffer A containing $50 \mathrm{~mm}$ Tris, $\mathrm{pH}$ 7.4, $150 \mathrm{~mm} \mathrm{NaCl}, 1 \%$ Nonidet P40, and protease inhibitors. Immunoprecipitation was performed as described previously (Zhang et al., 2007). To determine the formation of the kinesin-1/JIP1/Rab10 complex in vivo, postnatal day $0(\mathrm{P} 0)$ rat brains were homogenized in buffer $\mathrm{A}$. After clarification by centrifugation $\left(15,000 \times g\right.$ for $30 \mathrm{~min}$ at $\left.4^{\circ} \mathrm{C}\right)$, the supernatants were incubated with indicated antibodies or control IgGs for $2 \mathrm{~h}$ at $4^{\circ} \mathrm{C}$ and then incubated with protein $\mathrm{G}$ agarose beads overnight at $4^{\circ} \mathrm{C}$. Beads were washed three times with buffer $\mathrm{A}$, and the proteins 
bound to beads were eluted by boiling in $2 \times$ SDS loading buffer and were analyzed by immunoblotting with indicated antibodies.

The guanine nucleotide-selective binding assay was performed according to a previous report (Arimura et al., 2009). Briefly, affinitypurified hexahistidine (His6)-Rab10 $(2 \mu \mathrm{g})$ was incubated with $30 \mu \mathrm{M}$ GTP $\gamma S$ (a nonhydrolyzable GTP analog) or GDP for $1 \mathrm{~h}$ at $30^{\circ} \mathrm{C}$ in buffer $\mathrm{B}$ (25 mM Tris/HCl, pH 7.5, $10 \mathrm{~mm}$ EDTA, $1 \mathrm{~mm}$ DTT, and $5 \mathrm{~mm} \mathrm{MgCl}_{2}$ ) before incubating with GST-JIP1 $(5 \mu \mathrm{g})$ immobilized on glutathioneSepharose beads for $1 \mathrm{~h}$ at $30^{\circ} \mathrm{C}$. Next, beads were washed at least three times with buffer $\mathrm{B}$ and subjected to immunoblotting.

Immunostaining. Cultured neurons were fixed with $4 \%$ paraformaldehyde in PBS at room temperature (RT) for $20 \mathrm{~min}$ and permeabilized with $0.1 \%$ Triton X-100 in PBS for $10 \mathrm{~min}$. After extensive washing, neurons were blocked in PBS supplemented with $10 \%$ goat serum at RT for $1 \mathrm{~h}$, followed by incubation with primary antibodies overnight at $4^{\circ} \mathrm{C}$, washes, and incubation with Alexa Fluor 488-, Alexa Fluor 555-, or Alexa Fluor 647-conjugated secondary antibodies for $2 \mathrm{~h}$ at RT.

Immunohistochemical staining of brain sections was performed following a free-floating protocol. Briefly, brain sections were incubated with primary antibodies overnight at $4^{\circ} \mathrm{C}$ after treatment with $0.3 \%$ Triton X-100 and 10\% goat serum in PBS at RT for $1 \mathrm{~h}$, followed by three washes and then incubation with Alexa 488-, Alexa 555-, or Alexa 647conjugated secondary antibodies for $2 \mathrm{~h}$ at RT, and finally counterstained with DAPI for $10 \mathrm{~min}$ at RT before imaging.

Neuron culture, transfection, and imaging. The culture of cortical or hippocampal neurons was performed as described previously (Chen et al., 2006). Neurons were electroporated with the indicated plasmids using the Amaxa Nucleofector device before plating. In some experiments, testing plasmids were cotransfected with pEGFP-N1 plasmid at a ratio of 4:1. Neurons were cultured on coverslips coated with PDL. After culturing for $4 \mathrm{~h}$, the medium was changed to Neurobasal medium supplemented with $1 \%$ glutamate and $2 \%$ B27.

To quantify neuronal polarity, cultured hippocampal neurons were fixed $72 \mathrm{~h}$ after plating, and were stained with antibody against axonal marker Smi312. The images of neurons were captured with the Neurolucida system (Nikon). Neurites positive for Smi312 and longer than 100 $\mu \mathrm{m}$ were considered as axons.

For the analysis of subcellular localization, neurons were cotransfected with testing plasmids and pEGFP at a ratio of 4:1, then fixed and stained with indicated antibodies. Images were captured using a Nikon A1R inverted confocal microscope with $60 \times$ Plan Apo objective lens [numerical aperture (NA) 1.4 oil-immersion). To plot the relative immunofluorescence intensity of Rab10 in axons, acquired images were analyzed using ImageJ software. Cotransfected GFP was used to mark neuronal morphology.

Live cell imaging. Dissociated neurons were transfected with TDRab10 together with indicated plasmids. At $3 \mathrm{~d}$ in vitro (DIV3), cultures were switched to phenol red-free Neurobasal medium and maintained in an incubator at $37^{\circ} \mathrm{C}$ with $5 \% \mathrm{CO}_{2}$ during imaging process. Time-lapse images were acquired by capturing frames every $2 \mathrm{~s}$ continuously for 15 min using Nikon A1R inverted confocal microscope with $60 \times$ Plan Apo objective lens (NA 1.4 oil-immersion). Kymographs of TD-Rab10 vesicles were generated by ImageJ with a NeuronJ plugin.

In utero electroporation. In utero electroporation was performed as described previously (Saito and Nakatsuji, 2001; Wang et al., 2011). Briefly, targeting plasmids [siJIP1, scrambled siRNA (siScr), JIP1-M, or pCS2 vector] were mixed with pCAG-internal ribosomal entry site (IRES)-enhanced yellow fluorescent protein (EYFP) vector at a 4:1 ratio (in micrograms) in fast green and injected (1-2 $\mu$ l) into the lateral ventricles of the SD rat embryonic brains at embryonic day 15.5 (E15.5) of either sex. A pair of forceps electrodes was placed at the head position of each embryo and five square wave pulses $(60 \mathrm{~V}, 50 \mathrm{~ms})$ with an interval of $1 \mathrm{~s}$ (ECM830; BTX) were applied to the embryonic brains. At appropriate time points after electroporation, rat brains were dissected and fixed in 4\% paraformaldehyde in PBS for tissue sectioning and immunostaining. Neuronal morphology was traced and analyzed by ImageJ with the Simple Neurite Tracer plugin.
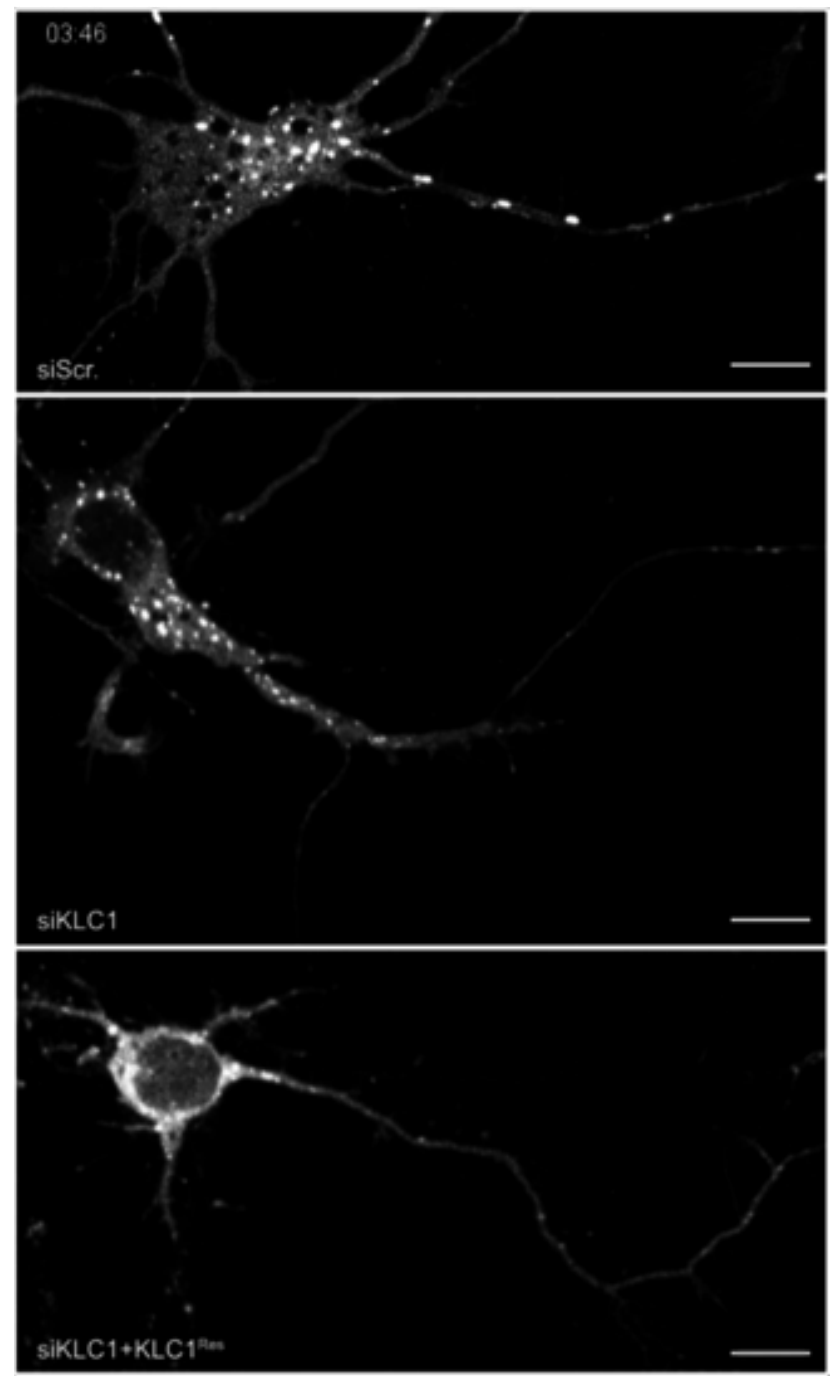

Movie 1. Live imaging of TD-Rab10-positive vesicles in the axons of DIV3 hippocampal neurons transfected with siScr or siKLC1, either alone or together with $\mathrm{KLC}^{\text {Res }}$. Scale bar, 10 $\mu \mathrm{m}$.

\section{Results}

Anterograde transport of Rab10-positive PPVs is mediated by kinesin-1

Recently, we have shown that membrane addition mediated by the insertion of Rab10-positive PPVs into specific sites of plasma membrane is critical for neuronal polarization and axonal growth. However, the mechanism underlying the directional trafficking of PPVs remains largely unknown. The combined observation of the selective positioning of kinesin-1 in nascent axons and the role of Rab10 in axon development inspired us to investigate whether anterograde transport of Rab10-positive PPVs in axons is mediated by kinesin-1 motor protein.

To study the role of kinesin-1 in anterograde transport of Rab10-positive PPVs, we generated a specific siRNA targeting against rat KLC1 (siKLC1), and determined its efficacy in cultured cortical neurons. As shown in Figure $1 A$, transfection with siKLC1, but not siScr, markedly downregulated the expression of endogenous KLC1. Then we monitored the displacement of TDRab10-containing vesicles in axons by time-lapse fluorescence microscopy (Fig. 1B; Movie 1), and found that downregulation of $\mathrm{KLC} 1$ caused a marked decrease in the percentage of the antero- 
A

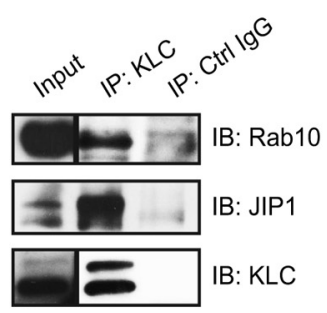

C

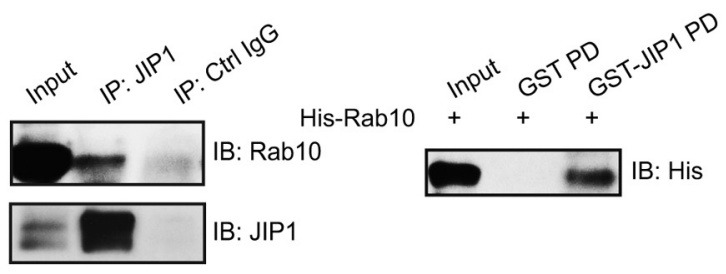

E

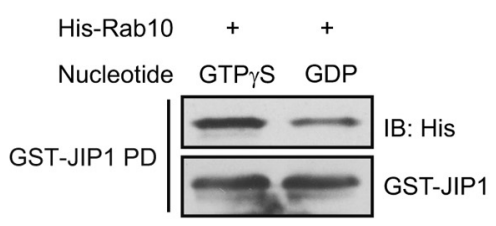

$\mathrm{H}$
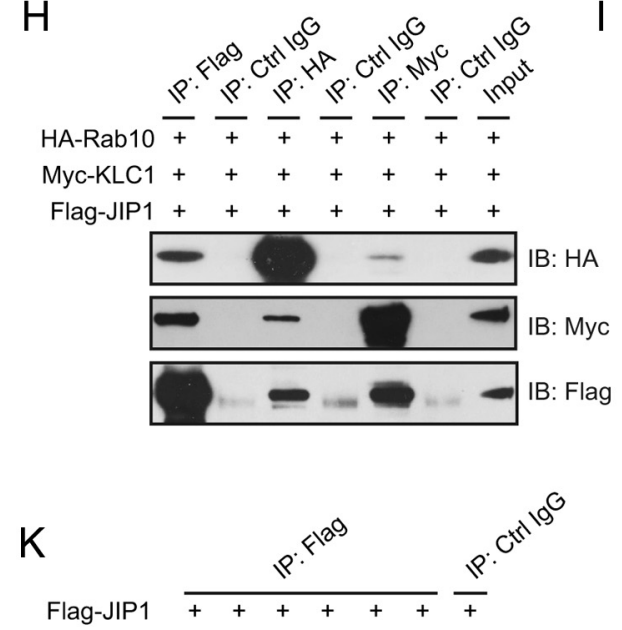

$\begin{array}{llllllll}\text { HA-Rab } & 5 a & 8 a & 8 b & 10 & 11 & 13 & 10\end{array}$

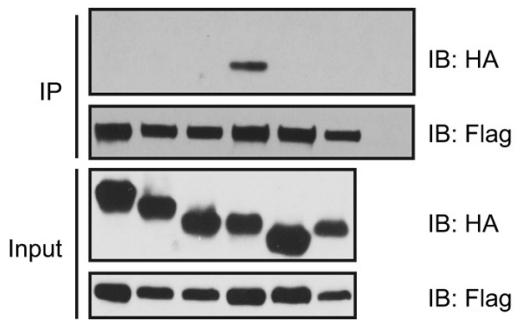

F HA-Rab10 WT Q68L

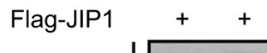

IP: HA

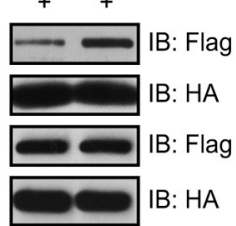

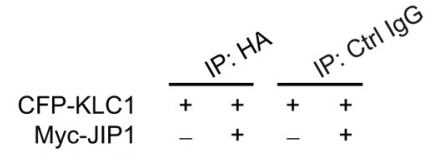

HA-Rab10 + + + +

IP

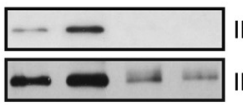

IB: GFP (low)

IP

Input

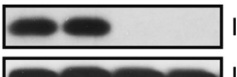

IB: GFP (high)

IB: $\mathrm{HA}$

IB: GFP

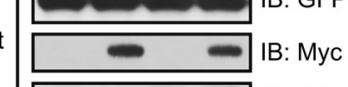

IB: HA

G $\quad$ HA-Rab10 WT Q68L

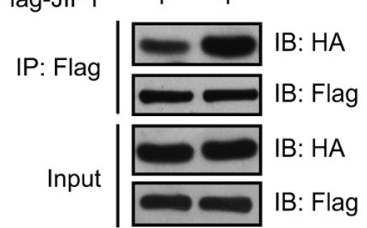

J

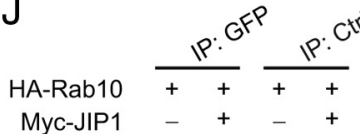

CFP-KLC1 ++++

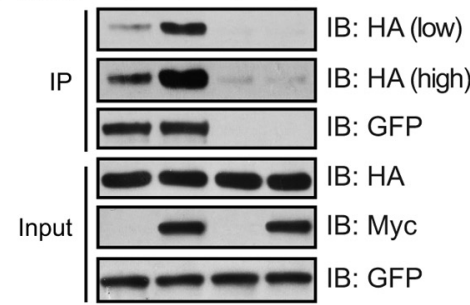

Figure 2. Rab10 associates with KLC via JIP1. A-C, Association of Rab10, JP1, and KLC in the rat brain. Homogenates of P0 rat brains were incubated with indicated antibodies. The same amount of control lgG (Ctrl lgG) was used as the control. Resulting immunocomplexes were subjected to IB with indicated antibodies. D, Purified His6-Rab10 (2 $\mu \mathrm{g})$ was incubated with glutathioneSepharose beads immobilized with the same amount of GST or GST-JIP1 (5 $\mu \mathrm{g})$. Bound proteins were subject to IB with anti-His antibody. E, Purified His6-Rab10 (2 $\mu \mathrm{g})$ preloaded with GTP $\gamma$ S or GDP was incubated with beads coupled with GST-JIP1 $(5 \mu \mathrm{g})$. Bound proteins were subjected to IB with anti-His or GST antibody. F, G, Lysates of HEK293 cells transfected with Rab10 (WT or Q68L form) and Flag-JIP1 were subjected to IP and IB with indicated antibodies. $\boldsymbol{H}$, Formation of the KLC1/JIP1/Rab10 complex in HEK293 cells transfected with indicated constructs. I, J, JIP1 enhances Rab10 association with KLC1 in HEK293 cells. Low and high exposures were used to display differences in band intensity, in the absence and presence of JIP1 expression. $K, L$, Cell lysates from HEK293 cells transfected with Flag-JIP1 and HA-Rabs were subjected to IP and then IB with anti-Flag or HA antibody. Note that only Rab10 is associated with JIP1.

gradely (from soma to neurite tips) transported vesicles and a corresponding increase of immobile vesicles (Fig. 1C; Movie 1). Notably, in siKLC1 neurons that exhibited an obvious axon, a large fraction of TD-Rab10 accumulated in the axon hillock and failed to be transported distally (Movie 1). As a consequence, the mean velocity of vesicle movement in axons also declined in siKLC1-transfected neurons, compared with control cells (Fig. $1 D)$. The above defects in anterograde transport of TD-Rab10 vesicles caused by siKLC1 were prevented by coexpression of
$\mathrm{KLC} 1^{\text {Res }}$, the siRNA-resistant form of KLC1 (Fig. 1A-D; Movie 1), thus excluding the potential off-target effects of siKLC1. These results suggest that kinesin-1 mediates the anterograde transport of Rab10-positive PPVs in the nascent axons of cultured neurons.

\section{Rab10 associates with KLC via JIP1}

Molecular motors load the membrane organelles directly or indirectly via adaptor proteins. Coimmunoprecipitation (co-IP) 
A

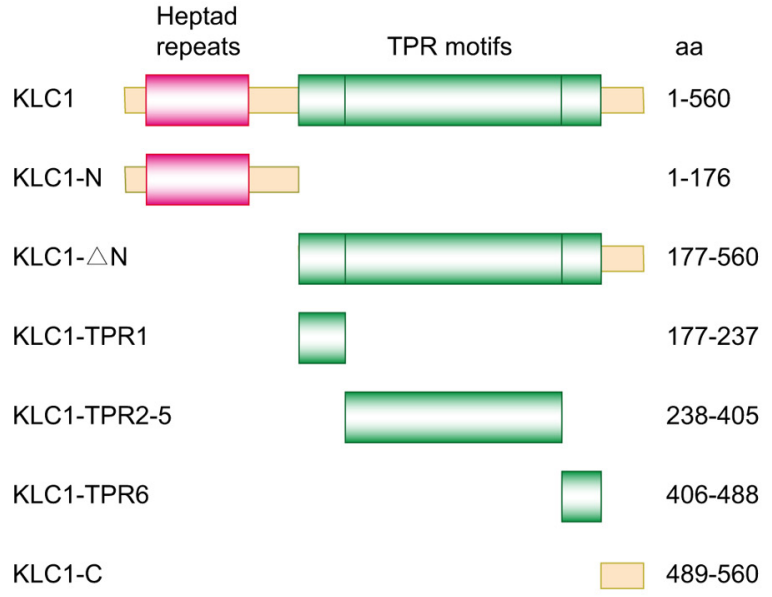

C

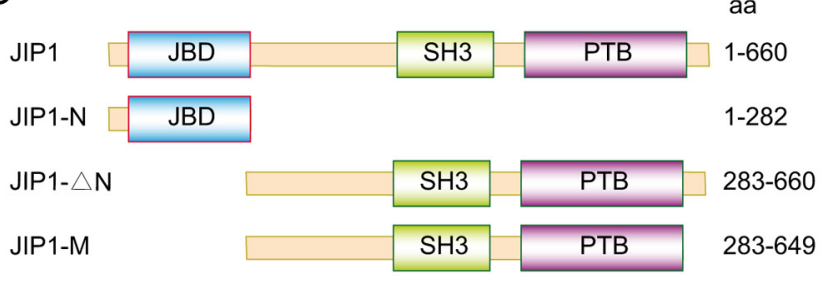

D
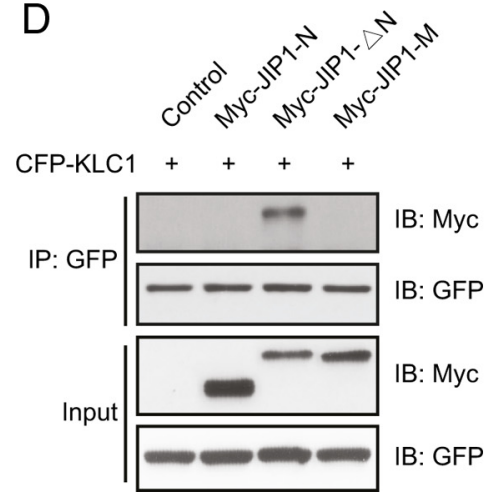

$E$
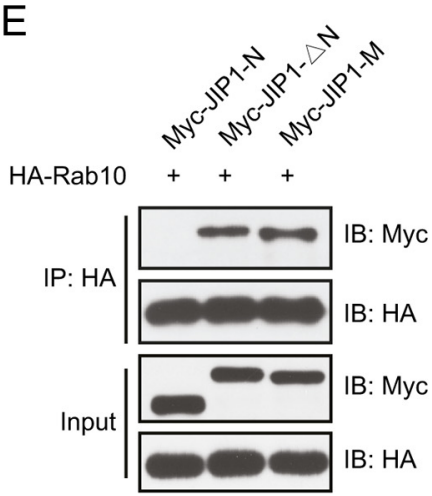

B

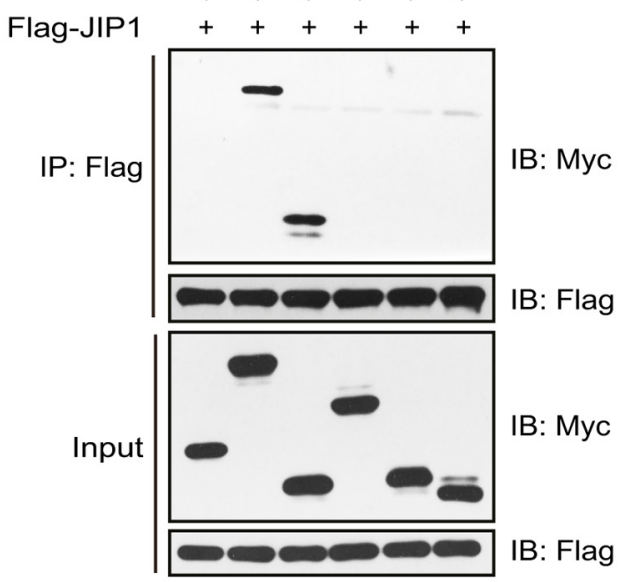

$\mathrm{F}$

\begin{tabular}{l|cc} 
& Interaction with KLC1 & Interaction with Rab10 \\
\hline JIP1 & + & + \\
JIP1-N & - & - \\
JIP1- $\triangle N$ & + & + \\
JIP1-M & - & +
\end{tabular}

Figure 3. Mapping of the KLC1 and JIP1 domains mediating the formation of the KLC1/JIP1/Rab10 complex. $\boldsymbol{A}$, Schematic structures of KLC1 full-length and various fragments. $\boldsymbol{B}$, Lysates of HEK293 cells transfected with Flag-JIP1, together with Myc-tagged KLC1 fragments were subjected to IP with anti-Flag antibody, and then IB with anti-Myc or Flag antibody. C, Schematic structures of JIP1 full-length and various fragments. D, Lysates of HEK293 cells transfected with CFP-KLC1, together with control plasmid or that encoding indicated Myc-tagged JIP1 fragments were subjected to IP with antibody against GFP, and then IB with Myc or GFP antibody. $E$, Lysates of HEK293 cells transfected with HA-Rab10 and Myc-tagged JIP1 domains were subjected to IP with antibody against HA, and then IB with Myc or HA antibody. F, Summary of interactions between JIP1 fragments and KLC1 or Rab10. G, Effects of JIP1 fragments on KLC1 association with Rab10. HEK293 cells were cotransfected with CFP-KLC and HA-Rab10, together with vehicle vectors or that encoding various JP1 fragments. Cell lysates were subjected to IP and then IB with indicated antibodies.

experiments showed that KLC and Rab10 formed a complex in the rat brain (Fig. $2 A, B$ ). Interestingly, JIP1 was also present in this complex (Fig. $2 A-C$ ). The Rab10 interaction with JIP1 is direct, because beads coupled with GST-JIP1 fusion protein, but not GST alone, could pull down His6-tagged Rab10 (Fig. 2D). Notably, GST-JIP1 pulled down more Rab10 preloaded with GTP $\gamma S$ than that preloaded with GDP (Fig. $2 E$ ), indicating that JIP1 favors the active form of Rab10. In line with this notion, co-IP experiments showed that Flag-JIP1 expressed in HEK293 cells preferentially interacted with Rab10 ${ }^{\mathrm{Q} 68 \mathrm{~L}}$, the GTP-locked active form of Rab10, compared with wild-type Rab10 (Fig. $2 F, G)$. Given that JIP1 is a typical adaptor linking kinesin-1 to cargos, we speculated that Rab10 might associate with KLC via
JIP1. To test this possibility, we determined the association between Rab10 and KLC under conditions with increased levels of JIP1. As shown in Figure 2H, when coexpressed in HEK293 cells, ectopic Rab10, JIP1, and KLC1 formed a ternary complex. Interestingly, the association between KLC1 and Rab10 was increased in cells with JIP1 overexpression (Fig. $2 I, J$ ). Thus, JIP1 links Rab10 to KLC. Among >60 mammalian Rabs, Rab5a, Rab8a, Rab8b, Rab11, and Rab13 have high homology with Rab10, and, of note, none of them was associated with JIP1 when coexpressed in HEK293 cells (Fig. $2 K, L$ ). This result indicates the specificity of the JIP1-Rab10 interaction.

To further investigate the role of kinesin-1/JIP1 complex in transporting Rab10 vesicles, we mapped domains of KLC1 and 
A

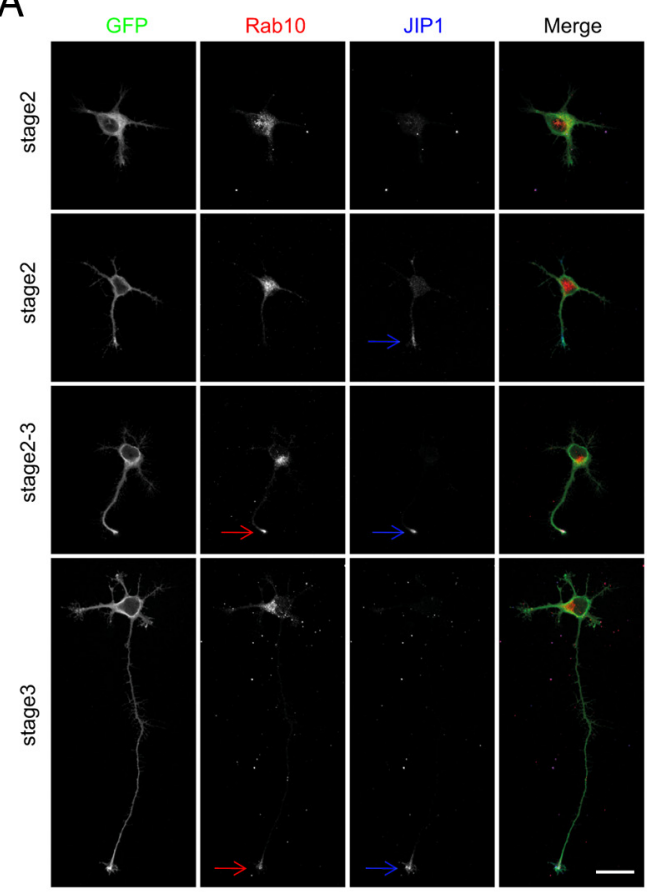

$\mathrm{H}$

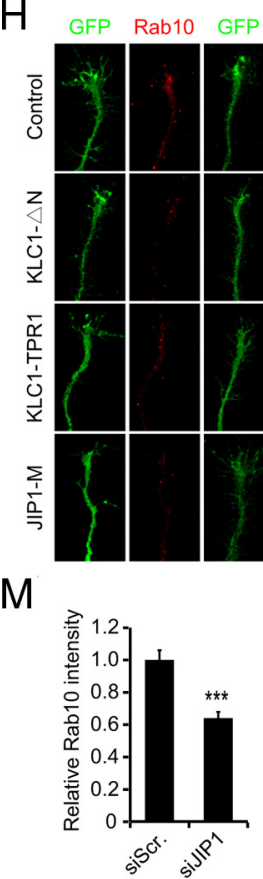

$\mathrm{N}$

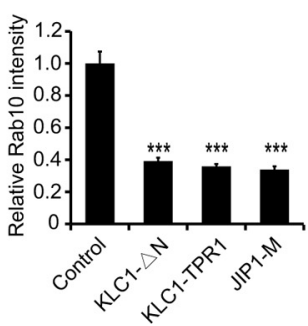

B

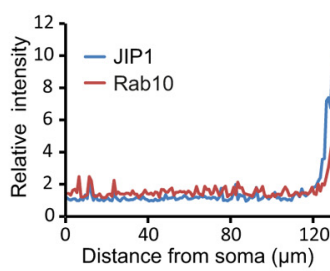

C
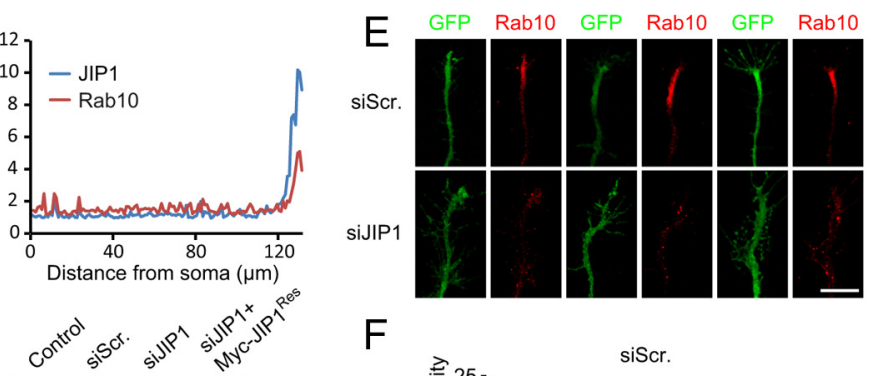

F

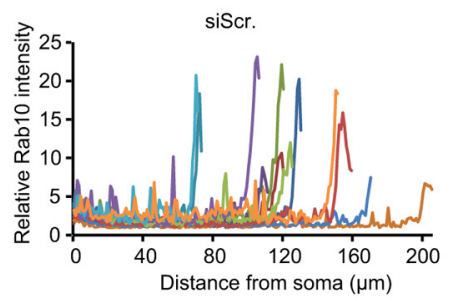

G

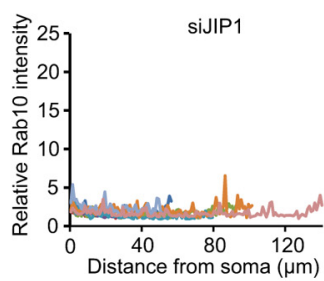

$\mathrm{J}$

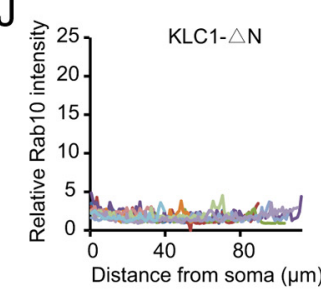

K

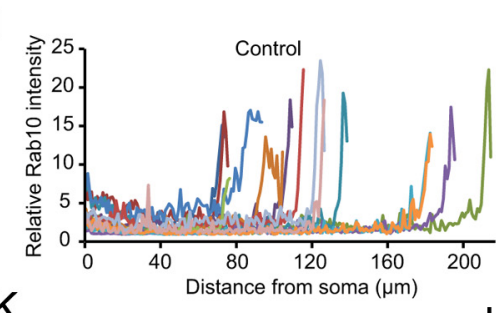

$\mathrm{L}$
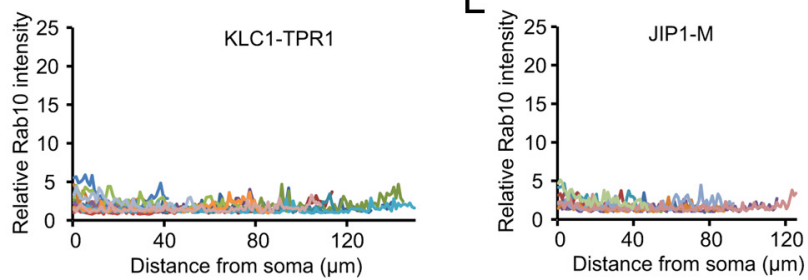

Figure 4. JIP1 interaction with KLC regulates the localization of Rab10 in axonal tips. A, GFP-transfected hippocampal neurons at different stages were stained for Rab10 and JIP1. Scale bar, 20 $\mu \mathrm{m} . \boldsymbol{B}$, Relative immunofluorescence intensity of Rab10 and JPP1 along the axon of a hippocampal neuron at stage 3. C, Cortical neurons were transfected with siScr, siJIP1, or siJIP1 plus Myc-JIP1 Res, the siRNA-resistant form of JIP1. Cell lysates were blotted with anti-JIP1 antibody, with actin and GAPDH as loading controls. D, Cortical neurons were transfected with CFP-KLC and HA-Rab10, together with siScr or siJIP1 constructs. Cell lysates were subjected to IP with antibody against HA, followed by IB with indicated antibodies. Note the almost complete disruption of the KLC1-Rab10 association under the condition with JIP1 knockdown. E, DIV3 hippocampal neurons transfected with siScr or siJP1 were stained with anti-Rab10 antibody. Shown are representative images of the Rab10 signals at distal regions of axons or the longest neurites. Scale bar, $10 \mu \mathrm{m} . \boldsymbol{F}, \mathbf{G}$, Distributions of Rab10 along axons or longest neurites in siScr $(\boldsymbol{F})$ or siJlIP1 (G) neurons. Shown are plots of relative intensity of Rab10 in the axon compared with that of the soma. Each color line represents an example neuron. $\boldsymbol{H}$, Distributions of Rab10 in distal regions of axons or longest neurites of DIV3 hippocampal neurons transfected with indicated plasmids. Scale bar, $10 \mu \mathrm{m}$. I-L, Distributions of Rab10 along axons or longest neurites in DIV3 hippocampal neurons transfected with vehicle $(I)$, KLC1- $\Delta \mathrm{N}(\boldsymbol{J})$, KLC1-TPR1 $(\boldsymbol{K})$, or JP1-M $(\boldsymbol{L})$. Data are presented as the ratio of the Rab10 intensity along the neurite to that of the soma with each color line representing an example cell. $\boldsymbol{M}, \boldsymbol{N}$, Quantification for relative intensities of Rab10 at distal regions of axons or the longest neurites (10 $\mu \mathrm{m}$ from the tip) of hippocampal neurons transfected with indicated constructs. Data are presented as the mean \pm SEM. ${ }^{* *} p<0.001$, Student's $t$ test. 
A

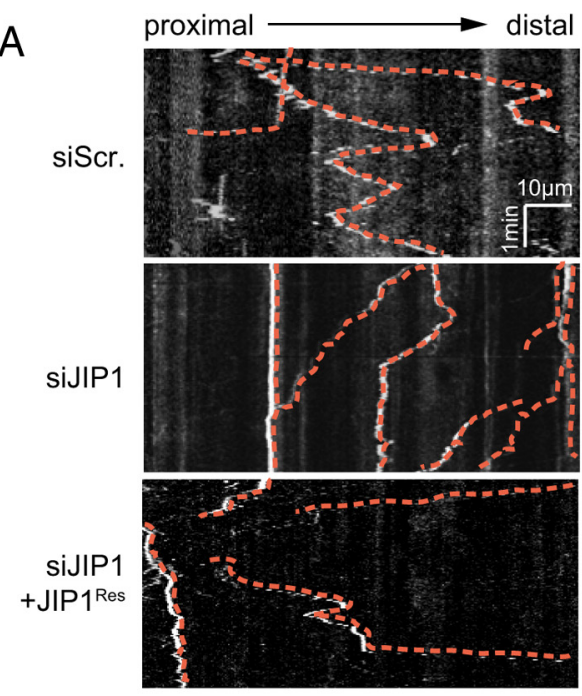

$\mathrm{D}$
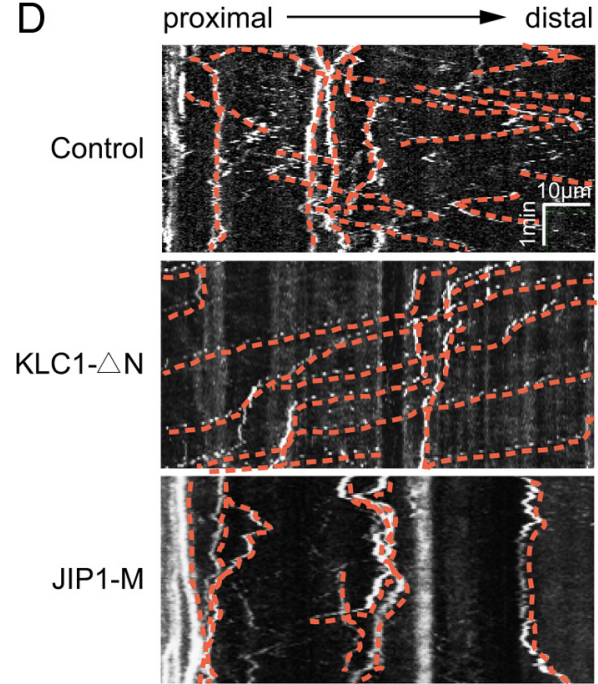

$\mathrm{B}$
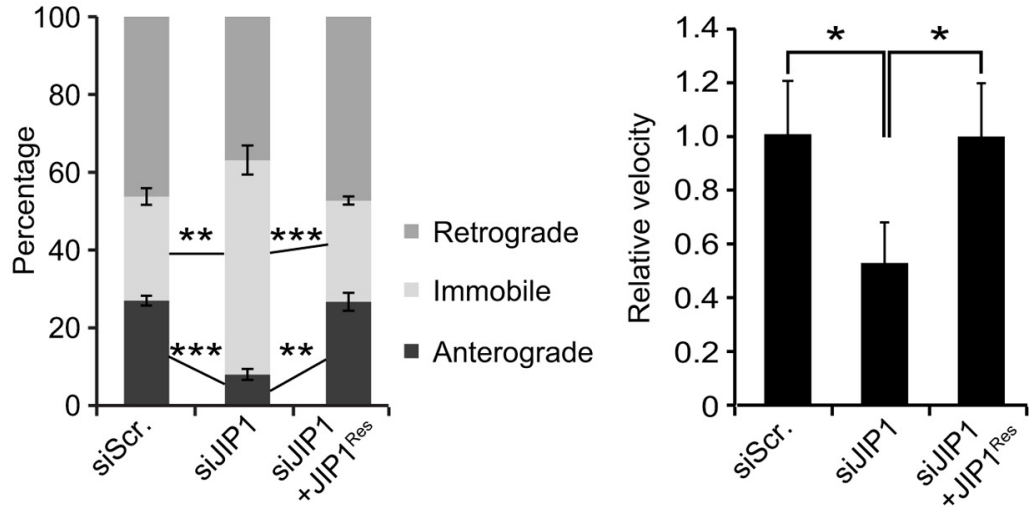

E

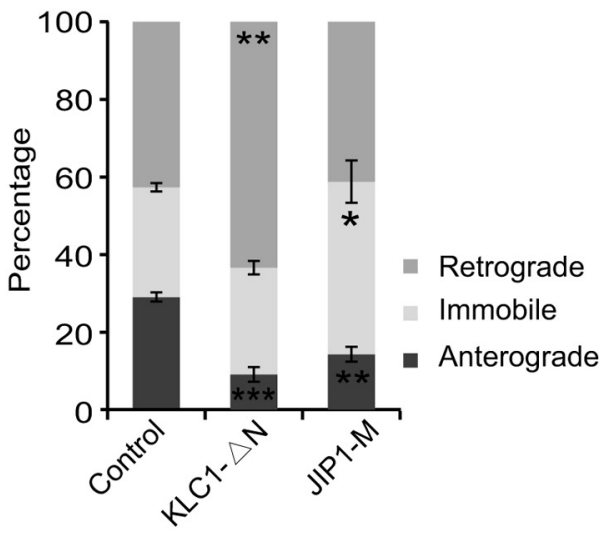

Figure 5. JIP1 mediates anterograde transport of Rab10 vesicles. A, Hippocampal neurons were transfected with TD-Rab10, together with siScr, siJIP1, or siJPP1 plus JIP1 Res. At DIV3, transportation of individual TD-Rab10 vesicles was recorded with live-imaging microscopy. Shown are representative kymographs of TD-Rab10 in axons or the longest neurites. Scale bar, $10 \mu \mathrm{m}$. B, Quantification for the percentage of anterograde, retrograde, or immobile TD-Rab10 vesicles for axons or longest neurites, in transfected hippocampal neurons. Data are presented as the mean \pm SEM. ${ }^{* *} p<0.01,{ }^{* * *} p<0.001$, Student's $t$ test. C, Quantification for the mean velocity of TD-Rab10 vesicles in axons of hippocampal neurons of indicated groups. Data are presented as the mean \pm SEM. ${ }^{*} p<0.05$, Student's $t$ test. $\boldsymbol{D}$, Hippocampal neurons were transfected with TD-Rab10, together with vehicle plasmid or that encoding KLC1- $\Delta \mathrm{N}$ or JIP1-M, followed by live imaging at DIV3. Shown are representative kymographs of TD-Rab10 vesicles in axons or longest neurites. Scale bar, $10 \mu \mathrm{m}$. $\boldsymbol{E}$, Quantification for the percentage of anterograde, retrograde, and immobile TD-Rab10 vesicles. Data are presented as the mean \pm SEM. ${ }^{*} p<0.05,{ }^{* *} p<0.01,{ }^{* * *} p<0.001$, Student's $t$ test.

JIP1 that were responsible for mediating the formation of the triple complex. First, we mapped the KLC1 domains that interact with JIP1. Previous studies have shown that KLC1 binds to KIF via the $\mathrm{N}$-terminal heptad repeats and to JIP1 via the six tetratricopeptide repeats (TPRs; Verhey et al., 1998, 2001). To gain insights into the minimal sequence of KLC sufficient for its interaction with JIP1, we generated myc-tagged fragments of KLC1 (Fig. 3A). We found that the first TPR motif (TPR1, amino acids 177-237) was sufficient to bind JIP1 when expressed in HEK293 cells (Fig. 3B). JIP1 contains JNK binding domain (JBD) at the $\mathrm{N}$ terminus, and the extreme $\mathrm{C}$-terminal 11 aa are required for its interaction with KLC (Verhey et al., 2001). To map the JIP1 regions mediating the interaction with Rab10 or KLC, HEK293 cells were transfected with Myctagged JIP1 fragments (Fig. $3 C$ ) together with HA-Rab10 or CFP-KLC1, followed by co-IP analysis. We found that the JIP 1 fragment with the deletion of the $\mathrm{N}$ and $\mathrm{C}$ termini, hereafter referred to as JIP-M, associated with Rab10, but not KLC1 (Fig. $3 D-F$ ). Similar to full-length JIP1, JIP1- $\Delta$ N, the construct with deletion of the N-terminal JBD domain, is also associated with both KLC1 and Rab10 (Fig. 3D-F). To this end, we have mapped the KLC1 and JIP1 domains that mediate the formation of the KLC/JIP1/Rab10 complex. Based on the information above, we screened dominant-negative constructs of JIP1 that could disrupt the coupling of Rab10 with kinesin-1 motors. As shown in Figure 3G, overexpression of Myc-JIP1-M, which interacts with Rab10 but not KLC1, disrupted the association between KLC1 and Rab10 (Fig. 3G, lane 4 ), and thus can be used as a dominant-negative construct. Interestingly, overexpression of JIP1- $\Delta \mathrm{N}$, which contains both Rab10 and KLC binding regions, markedly increased the association between KLC1 and Rab10 (Fig. 3G, lane 3). This result indicates that JNK binding is dispensable for the role of JIP 1 in bridging the KLC1-Rab10 interaction. 

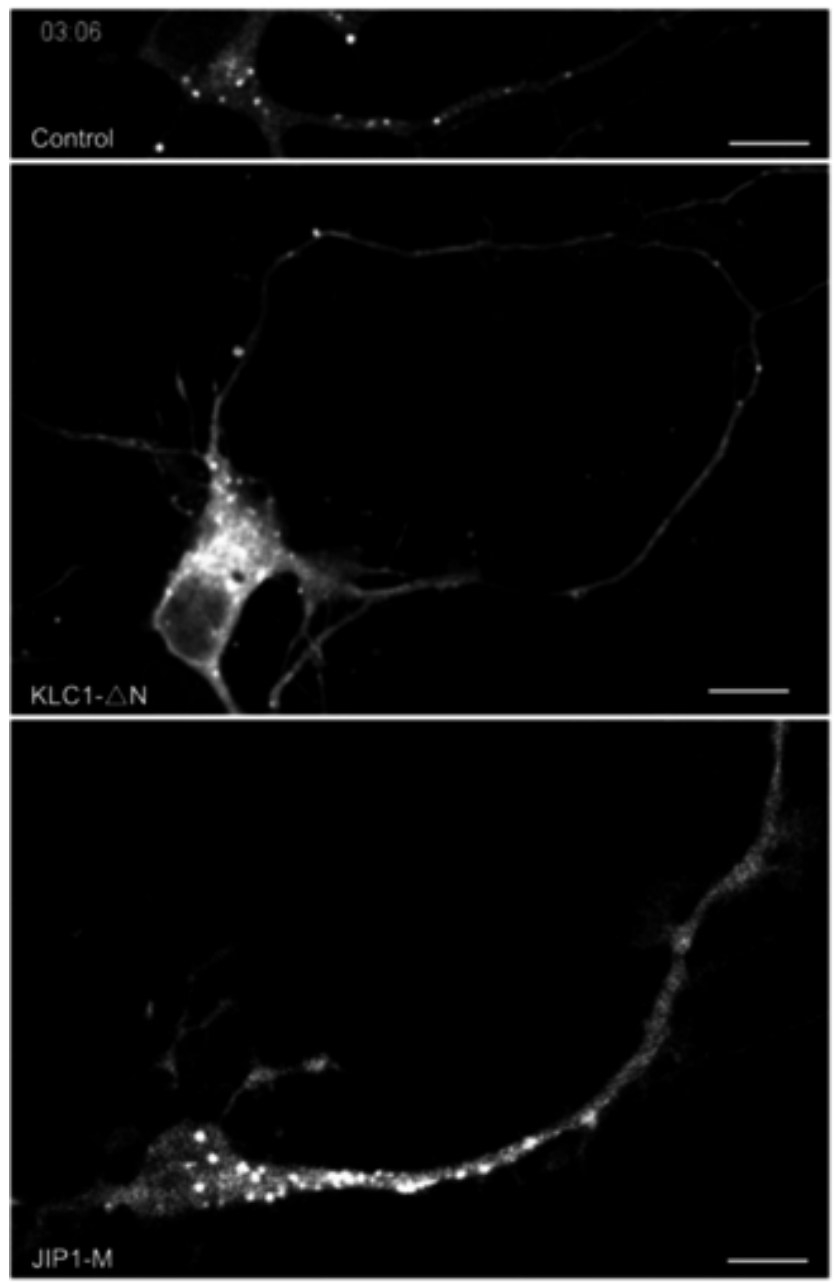

Movie 2. Live imaging of TD-Rab10-positive vesicles in the axons of DIV3 hippocampal neurons transfected with vehicle plasmid or that encoding KLC1- $\Delta \mathrm{N}$ or JIP1-M. Scale bar, 10 $\mu \mathrm{m}$.

JIP1 interaction with KLC regulates the localization of Rab10 in axonal tips

Having shown the physical composition of the KLC/JIP1/Rab10 complex, we next determined the role of this complex in axonal transport of Rab10 vesicles. First, we analyzed the spatialtemporal localization of JIP1 and Rab10 during axon development in cultured hippocampal neurons. In agreement with previous observations (Dajas-Balador et al., 2008; Wang et al., 2011), we found that both JIP1 and Rab10 are specifically enriched in nascent and mature axonal terminals of cultured hippocampal neurons (Fig. $4 A, B$ ). Interestingly, JIP1 localized to a single neurite of neurons at stage 2 before any of the processes were morphologically distinct (Fig. $4 A$, second row), and Rab10 began exhibiting polarized distribution during transition from stage 2 to stage 3 (Fig. $4 A$, third row from top). This pattern along with the identification of the KLC/JIP1/Rab10 complex prompted us to determine the role of JIP1 and its interaction with KLC or Rab10 in axonal localization of Rab10. After the screening of several sequences, a vector encoding an siRNA against rat JIP1 (siJIP1) was generated, and its effectiveness in suppressing JIP1 expression was shown in primary neurons. As shown in Figure $4 C$, transfection with the siJIP1 construct, but not that encoding a scrambled sequence (siScr), markedly decreased the expression of endogenous JIP1. The association between KLC1 and Rab10 was disrupted in cells with
JIP1 knockdown (Fig. 4D). In DIV3 hippocampal neurons transfected with siScr, Rab10 exhibited polarized distribution in distal axons (Fig. 4E, F). However, this pattern did not occur in any neurite of siJIP1-transfected neurons (Fig. $4 E, G$ ). The intensity of Rab10 in the tips of axons or the longest neurites in siJIP1 cells was much lower than that of control cells (Fig. 4M). These results suggest that JIP1 is required for polarized distribution of Rab10 during axon development. Similar to siJIP1, the overexpression of the dominant-negative mutant of KLC (KLC1- $\Delta \mathrm{N})$, KLC1-TPR1, or JIP1-M prevented the polarized distribution of Rab10 (Fig. $4 H-L, N$ ), most likely by disrupting the KLC/JIP1/Rab10 complex. Thus, JIP1 interaction with kinesin-1 motors determines the polarized distribution of Rab10 that is required for axon development.

\section{JIP1 and its interaction with KLC regulate anterograde transport of Rab10-positive vesicles}

Having shown the effects on Rab10 localization in fixed cells, we next determined the behavior of TD-Rab10 vesicles in living neurons after manipulation of the JIP1 level or the integrity of the KLC/JIP1/Rab10 complex. First, we monitored the movement of TD-Rab10 vesicles in the axon or the longest neurite of cultured hippocampal neurons transfected with siJIP1 or siScr (Fig. $5 A$ ). We found that the percentage of anterograde vesicles was significantly decreased and that of immobile vesicles was increased correspondingly in JIP1-depleted neurons (Fig. 5B). Notably, JIP1 knockdown had no apparent effect on the percentage of retrograde vesicles (Fig. 5B). JIP1 knockdown also caused a decrease in the velocity of TD-Rab10 vesicles (Fig. $5 C$ ). The effect of siJIP1 was specific, because cotransfection with the siRNAresistant form of JIP1 (JIP1 ${ }^{\text {Res }}$; Fig. $4 C$ ) prevented the changes in the behaviors of TD-Rab10 vesicles (Fig. $5 A-C$ ).

We also determined the effects of KLC1- $\Delta \mathrm{N}$ and JIP1-M on the transport of TD-Rab10 vesicles (Fig. 5D; Movie 2). Similar to siJIP1 or siKLC1, expression of JIP1-M also impaired anterograde transport of Rab10 vesicles, increased the ratio of immobile vesicles, and had no effect on that of retrograde vesicles (Fig. $5 E$ ). Notably, while the expression of KLC- $\Delta \mathrm{N}$ also decreased the frequency of anterograde transport of Rab10 vesicles, it led to a significant increase in the frequency of retrograde transported vesicles and had no effect on that of immobile vesicles (Fig. $5 E$ ). The enhancement of retrograde transport caused by KLC1- $\Delta \mathrm{N}$ might be due to the switch of motor proteins associated with JIP1 cargos, as shown in a recent report (Fu and Holzbaur, 2013). Together, these results support the conclusion that JIP1 mediates anterograde transport of Rab10 vesicles through association with kinesin-1 motors.

\section{JIP1 and the kinesin-1/JIP1/Rab10 complex are required for} neuronal polarization and axonal extension in cultured hippocampal neurons

We have shown recently that Rab10 vesicles are required for directional membrane addition during axon development (Wang et al., 2011; Liu et al., 2013). We predicted that the impairment in the anterograde transport of Rab10 cargos might affect axon specification and/or extension. Indeed, JIP1 has been shown to be required for axon development in cultured cortical neurons (Dajas-Balador et al., 2008). In agreement with this finding, transfection of hippocampal neurons with siJIP1 before plating resulted in a marked impairment in axon development at DIV3, compared with cultures transfected with control siRNA (i.e., siScr; Fig. $6 A, B)$. The reduction in axon development was reflected from the reduced percentage of neurons with a single axon (1 axon), which was positively stained with axonal marker 
A

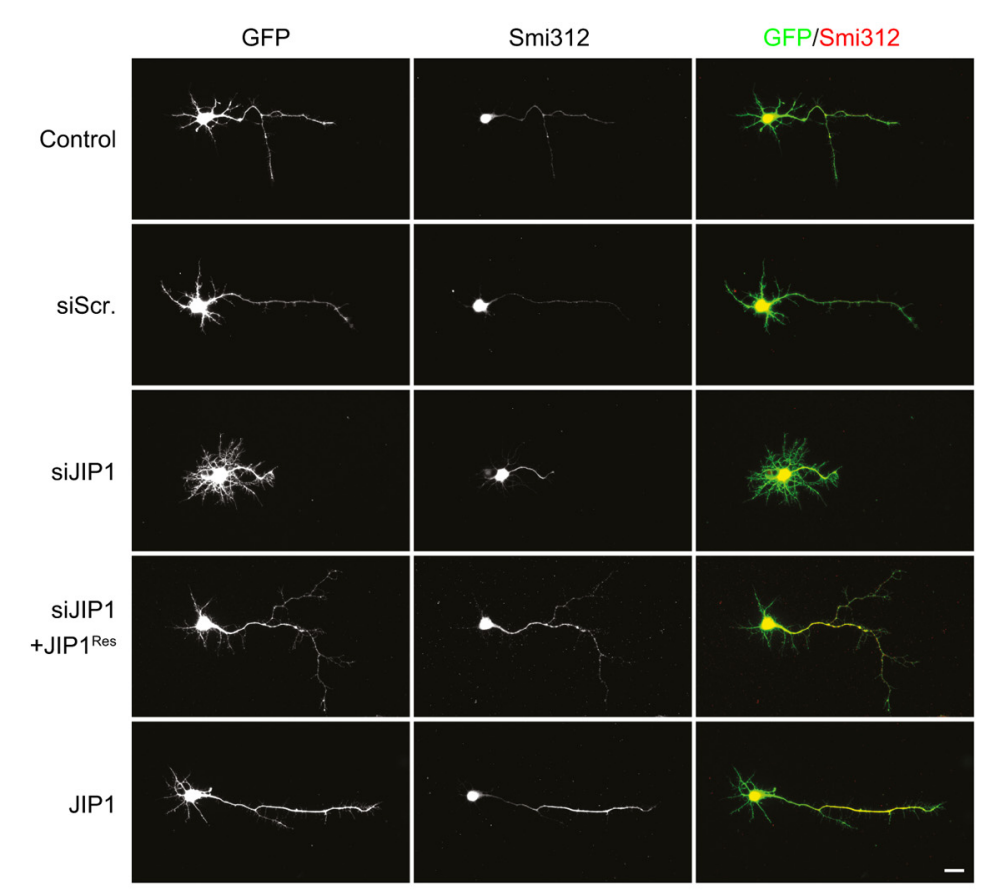

D

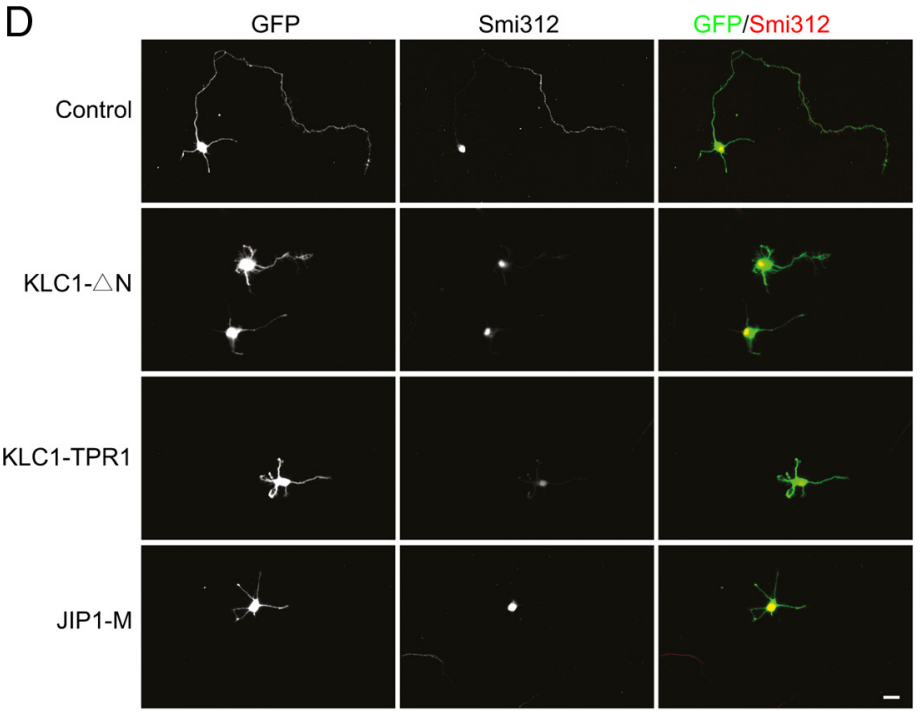

B
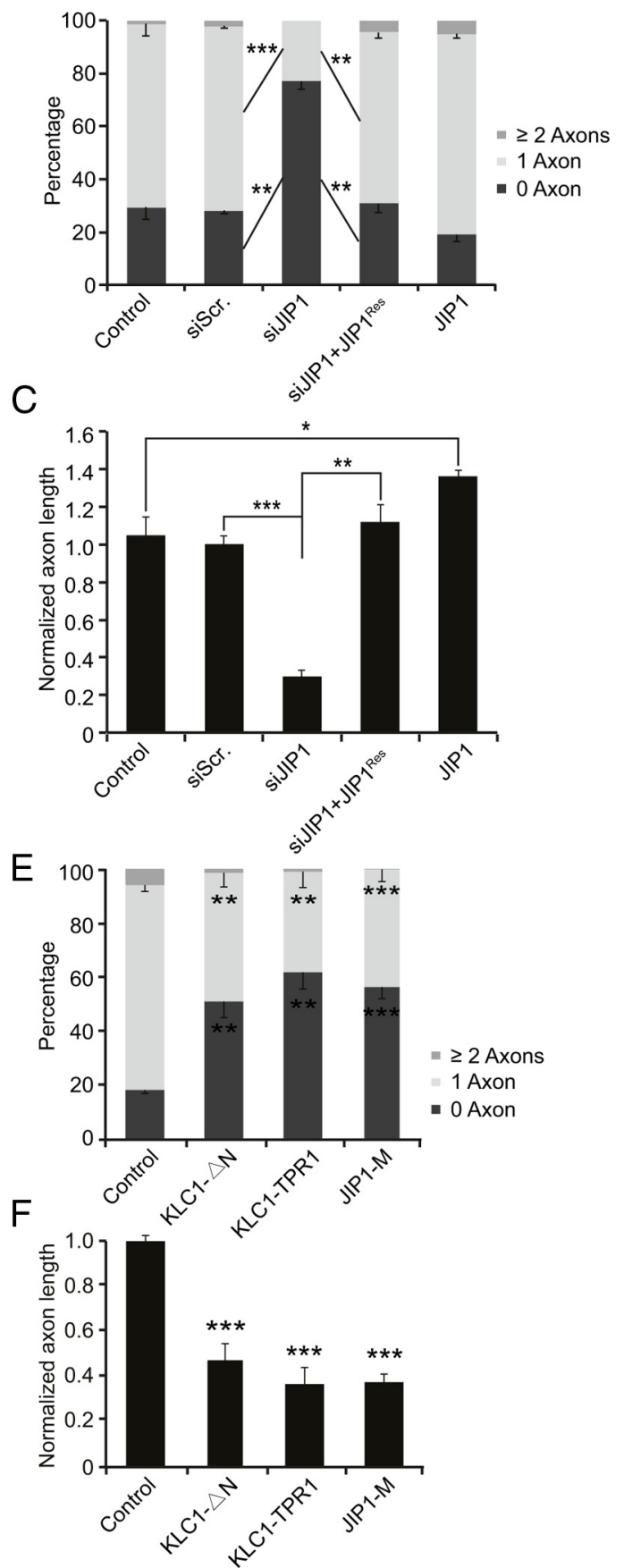

Figure 6. $\mathrm{KLC/JIP1/Rab10} \mathrm{complex} \mathrm{is} \mathrm{important} \mathrm{for} \mathrm{axon} \mathrm{development.} \boldsymbol{A}, \boldsymbol{D}$, Hippocampal neurons were transfected with indicated plasmids, together with EGFP, and then stained with Smi312 antibody at DIV3. Shown are representative images for neurons in each group. Scale bar, $20 \mu \mathrm{m} . \boldsymbol{B}, \boldsymbol{E}$, Quantification for the percentage of cells with different morphology (0 axon, neurons without axon; 1 axon, neurons with a single axon positive for Smi312 and longer than $100 \mu \mathrm{m} ; \geq 2$ axons, neurons with $>2$ axons). Data are presented as the mean \pm SEM. ${ }^{* *} p<0.01$, ${ }^{* * *} p<0.001$, Student's $t$ test. $\boldsymbol{C}, \boldsymbol{F}$, Quantification for average length of axons in each group. ${ }^{*} p<0.05,{ }^{* *} p<0.01$, ${ }^{* * *} p<0.001$, Student's $t$ test.

Smi312 and increased the percentage of neurons without axon $(0$ axon; Fig. $6 B$ ). In addition, the length of axons was significantly shorter in neurons with JIP1 knockdown (Fig. 6C). Interestingly, siJIP1 neurons exhibited many minor neurites with excessive branches (Fig. 6A, middle row), reminiscent of undirectional transport of membrane cargos. This result is in line with the role of JIP1 in anterograde transport of Rab10-positive PPVs. The effect of siJIP1 on axon development could be significantly prevented by the coexpression of JIP $1{ }^{\text {Res }}$ (Fig. $6 A-C$ ), thus excluding potential off-target effects of siRNA. Next, we determined the effect of increased JIP1 in cultured hippocampal neurons and found that the overexpression of JIP1 slightly increased axon length but had no effect on neuronal polarity (Fig. $6 A-C$ ). The amount of available endogenous cargos or motor proteins may limit the effect of ectopic JIP1.

We also determined the effects of dominant-negative mutants of KLC1 or JIP1 on neuronal polarity and axon development of cultured hippocampal neurons (Fig. $6 D-F$ ). As shown in Figure $6 E$, transfection with KLC1- $\Delta \mathrm{N}$, KLC1-TPR1, or JIP1-M caused impairment in the neuronal polarity of DIV3 neurons, as re- 


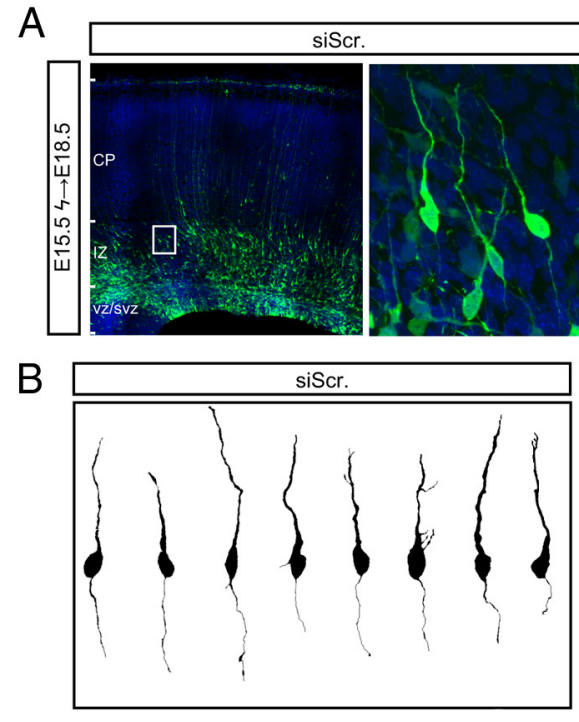

D

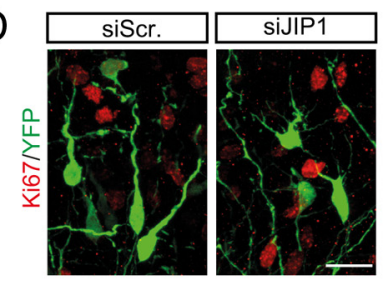

E

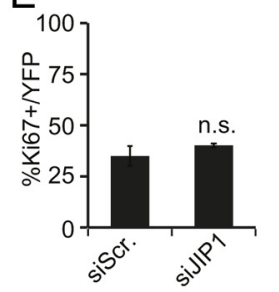

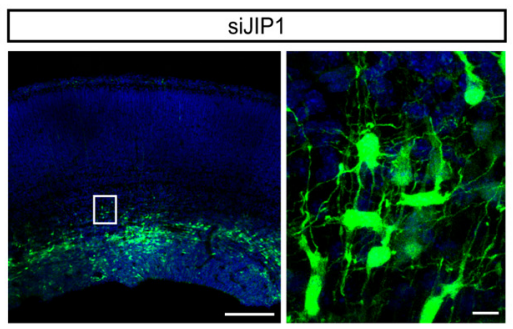

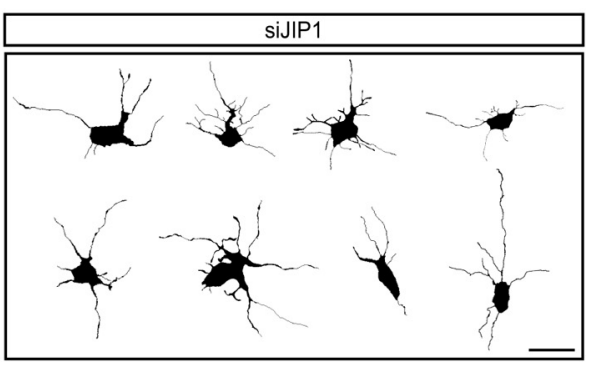

F

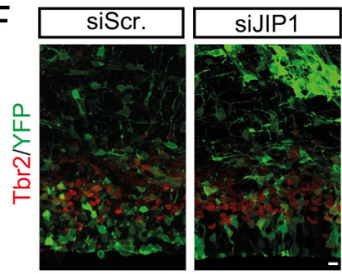

C

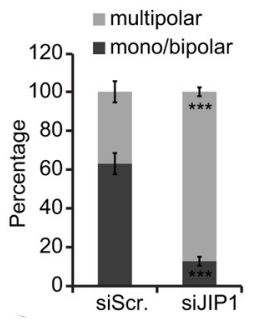

G

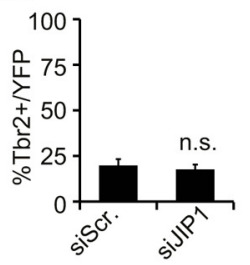

$\mathrm{H}$

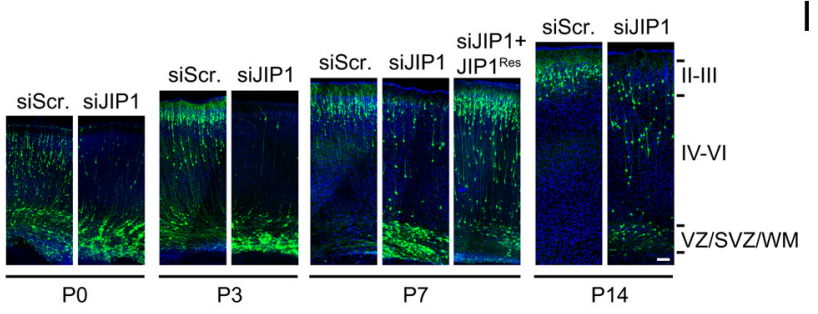

I
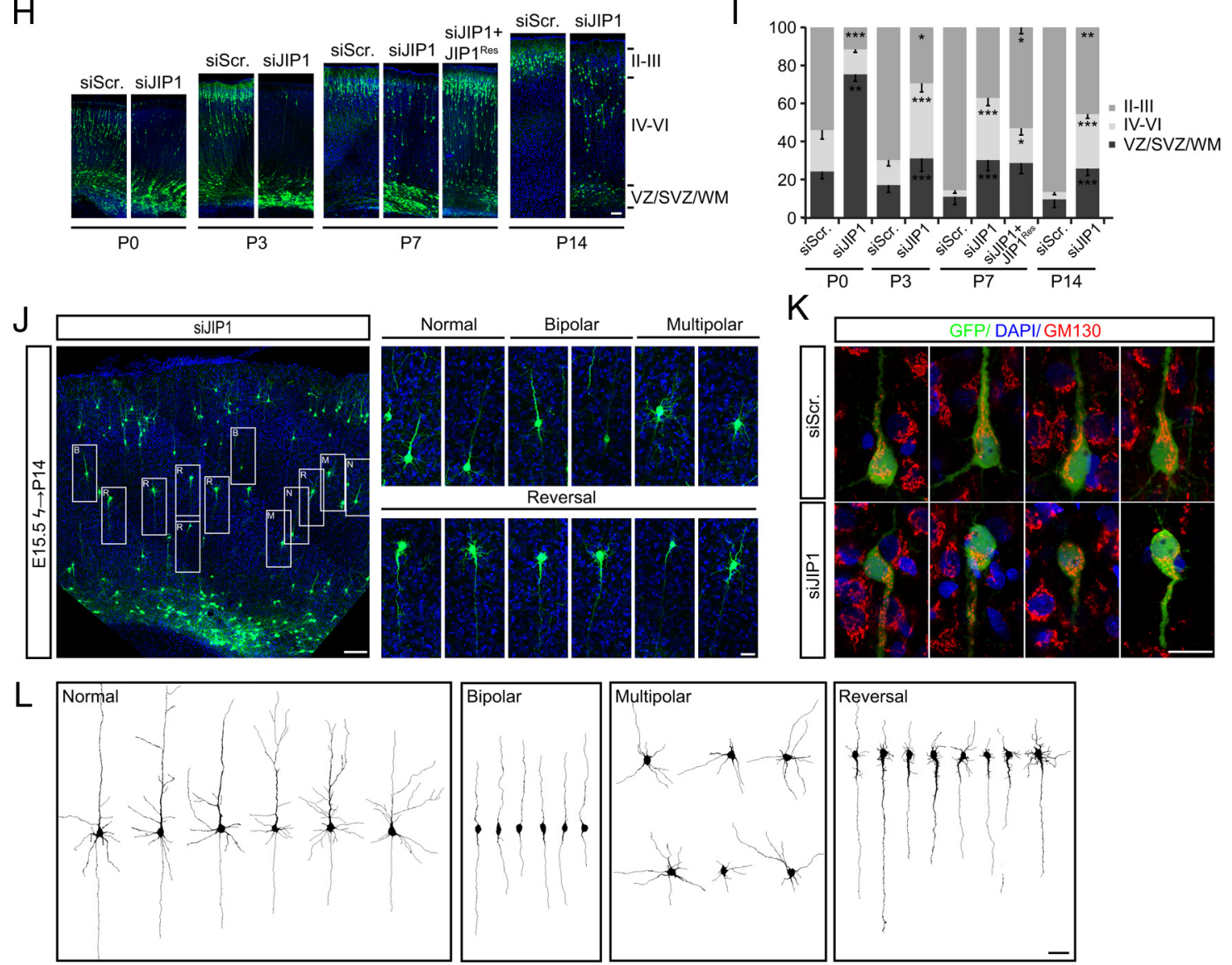

Figure 7. The Role of JIP1 in neocortical development in vivo. $\boldsymbol{A}$, Representative images of E18.5 neocortical slices from rat embryos, which were electroporated at E15.5 with plasmids encoding siScr or siJIP1, together with pCAG-EYFP. Scale bar, $200 \mu \mathrm{m}$. Enlarged areas show YFP-positive neurons in the IZ of siScr- or siJIP1-electroporated cortical sections. Scale bar, $10 \mu \mathrm{m}$. $\boldsymbol{B}$, Representative reconstructed images of YFP-positive cells in the IZ regions of electroporated cortical sections. Scale bar, $20 \mu \mathrm{m}$. $\mathbf{C}$, Quantification for the percentage of monopolar/bipolar and multipolar YFP-positive neurons in the SVZ and IZ regions of electroporated cortical sections. Data are presented as the mean \pm SEM. ${ }^{* * *} p<0.001$, Student's $t$ test. (Figure legend continues.) 
flected in the increase in neurons without axons and the decrease of neurons with a single axon (1 axon). Moreover, the length of axons was markedly decreased in KLC1- $\Delta \mathrm{N}$, KLC1-TPR1, or JIP1-M neurons (Fig. 6F). These results suggest an important role of the kinesin-1/JIP1/Rab10 complex in neuronal polarization and axon extension.

\section{JIP1 is required for neuronal migration and polarization of cortical neurons in vivo}

After the final division, the postmitotic neocortical pyramidal cells migrate along the radial glia following the inside-out pattern to form the cortical plate, which can be further divided into upper layers (layers II/III) and lower layers (layers IV-VI). During the radial migration, the neocortical pyramidal neurons experience a series of morphological maturation changes, including a transition from multipolar to monopolar/bipolar stages at the intermediate zone (IZ), followed by elongation of the trailing process that will later become the single axon, and then elaboration of dendritic arbors from leading processes (Nadarajah et al., 2001; Noctor et al., 2004; Barnes and Polleux, 2009). It is generally believed that the transition from the multipolar to monopolar/bipolar stages is a critical period for the establishment of neuronal polarity. To determine the role of JIP1 in polarization of newborn cortical neurons, we applied in utero electroporation to introduce siJIP1 or siScr construct, together with pCAG-IRES-EYFP into rat cortical progenitors at E15.5, when layer II/III neurons are born (Fame et al., 2011). The morphology of YFP-positive cells was analyzed $72 \mathrm{~h}$ after electroporation. In a control group transfected with siScr, $\sim 60 \%$ of the YFP-positive cells had finished the transition from the multipolar to monopolar/bipolar stages, and some of them already had migrated into the cortical plate (CP). In contrast, most JIP1 knockdown neurons ( $90 \%)$ remained at the multipolar stage and failed to migrate out of the subventricular zone (SVZ; Fig. $7 A-C$ ). To determine whether this delayed migration is due to the interference in the exit of the cell cycle or the differentiation of newborn neurons, we analyzed cell fate after electroporation by staining with antibodies against Ki67, which labels proliferating cells, or Tbr2, a T-domain transcription factor that marks intermediate progenitor cells for postmitotic projection neurons. We found that JIP1 knockdown had no effect on the percentage of either Ki67-positive cells or Tbr2-positive cells (Fig. 7D-G), suggesting that JIP1 unlikely affects differentiation of neuronal precursors in the SVZ.

Abnormal neuronal morphogenesis may affect cell positioning. We analyzed the effects of siJIP1 at different developmental stages and found that siJIP1 indeed markedly impaired neuronal

\section{$\leftarrow$}

(Figure legend continued.) $\quad \boldsymbol{D}, \boldsymbol{F}$, Cortical sections from rat embryos electroporated at E15.5 with EYFP together with indicated plasmids were stained with Ki67 antibody (D) or Tbr2 antibody $(\boldsymbol{F})$. Scale bars: $\boldsymbol{D}, 20 \mu \mathrm{m} ; \boldsymbol{F}, 10 \mu \mathrm{m}$. $\boldsymbol{E}, \boldsymbol{G}$, Quantification for the percentage of Ki67positive $(\mathrm{Ki} 67+; \boldsymbol{E})$ or Tbr2-positive (Tbr2 $+; \boldsymbol{G}$ ) cells among YFP-positive cells in VZ/SVZ/IZ regions of cortical sections. n.S., Not significant, Student's $\boldsymbol{t}$ test. $\boldsymbol{H}$, Representative images of rat neocortical slices from P0, P3, P7, or P14, after electroporation at E15.5. Scale bar, $100 \mu \mathrm{m} . I$, Quantification for the percentages of YFP-positive neurons in each cortical zone of rat neocortical slices at different stages. Data are presented as the mean \pm SEM. ${ }^{*} p<0.05,{ }^{* *} p<0.01$, ${ }^{* * *} p<0.001$, Student's $t$ test. J, P14 neocortical slices from rat embryos, which were electroporated at E15.5 with plasmids encoding siJIP1, together with PCAG-EYFP. Scale bar, $200 \mu \mathrm{m}$. Enlarged areas show YFP-positive neurons in layers IV-VI of siJIP1-electroporated cortical sections with various morphologies. Scale bar, $50 \mu \mathrm{m}$. $\boldsymbol{K}, \mathrm{P} 14$ neocortical slices from rat embryos electroporated at E15.5 with plasmids encoding siScr or siJIP1, together with pCAG-EYFP, were stained with GM130 antibody. Scale bar, $20 \mu \mathrm{m}$. $L$, Representative tracings of YFP-positive neurons in layers IV-VI of siJIP1-electroporated cortical sections. Scale bar, $50 \mu \mathrm{m}$. migration in every observation stage-P0, P3, P7, and P14 (Fig. $7 H)$. The migration defects were reflected from a decreased percentage of cells arriving at the CP and an increased percentage of cells accumulated in VZ/SVZ/WM (white matter) regions (Fig. $7 I)$. As indicated by an example and quantification for rats at P7, the migratory defect could be partially rescued by coexpression of JIP1 ${ }^{\text {Res }}$ (Fig. $7 \mathrm{H}, I$ ). The observed effects on neuronal migration caused by JIP1 downregulation are presumably attributed to defects in neuronal polarization.

Next, we analyzed single-cell morphology when JIP1 was downregulated. At P14, $~ 90 \%$ of control cells electroporated at E15.5 had already arrived at layer II/III (Fig. 7 H,I), with the morphology of a typical pyramidal neuron emanating a thick apical dendrite toward the pia and a thin axon toward the ventricle. Interestingly, more than half of siJIP1 neurons failed to arrive at upper layers and remained at lower layers or accumulated in VZ/SVZ/WM regions. We analyzed the morphology of single cells remained in layers IV-VI. Notably, we found that only a small fraction $(20.7 \pm 2.9 \%)$ exhibited normal pyramidal cell morphology, and some of them still remained at nascent intermediate stages-bipolar $(19.6 \pm 2.8 \%)$ or multipolar $(21.8 \pm$ $4.0 \%)$. More strikingly, a considerable fraction of them $(37.8 \pm$ $4.0 \%$ ) extended a thick process toward the ventricle, which is morphologically similar to apical dendrites (Fig. $7 \mathrm{~J}, L$ ). Notably, most $(70.1 \pm 6.4 \%)$ of these apical dendrite-like processes contained the cis-Golgi marker GM130 (Fig. $7 \mathrm{~K}$ ), which has been shown to be localized in leading processes that are destined to become apical dendrites (Horton et al., 2005). To our knowledge, this type of reversal of neuronal polarity has never been reported before, and might be the result of the mistargeting of membrane cargos due to JIP1 knockdown.

\section{Blockade of KLC/Rab10 association by JIP1-M inhibits neuronal polarity and axon development in vivo}

Using the above approaches, we also determined the effect of JIP-M, whose expression was able to disrupt the association of Rab10 with KLC1. Similar to siJIP1, electroporation of JIP1-M also inhibited the morphological transition of newborn cortical neurons from multipolar to monopolar/bipolar stages during crossing of the IZ regions (Fig. $8 A-C$ ), without affecting precursor proliferation and neuronal fate determination (Fig. $8 D-G$ ). In addition, the expression of JIP1-M slightly affected neuronal migration at the embryonic stages. However, the defects in neuronal migration were not seen in postnatal rats (Fig. $8 H$ ). Given that upper layer cortical neurons send out callosal axons that form the corpus callosum, one of the main axonal tracts linking two hemispheres (Mitchell and Macklis, 2005; Fame et al., 2011), we determined the effects of JIP1-M on the development of callosal axons from P3 rats electroporated at E15.5. The development of callosal axons was evaluated by the following two parameters: (1) the fluorescence intensity of axon bundles in the midline and contralateral side of the brain; and (2) the distance from midline to the forefront of the contralateral axonal tracts. As shown in Figure 8, $H$ and $I$, labeled callosal axons in control animal at $\mathrm{P} 3$ had crossed the midline and extended to the contralateral side of the cortex, whereas such axon projection was severely impaired in JIP1-M-transfected animals. Quantitatively, the intensity of YFP signals at the midline and the distance from midline to the end of axonal tracts were markedly decreased in the JIP1-M group compared with the vehicle control group (Fig. $8 J, K)$. These results suggest that JIP1 interaction with Rab10 is important for callosal axon extension. 

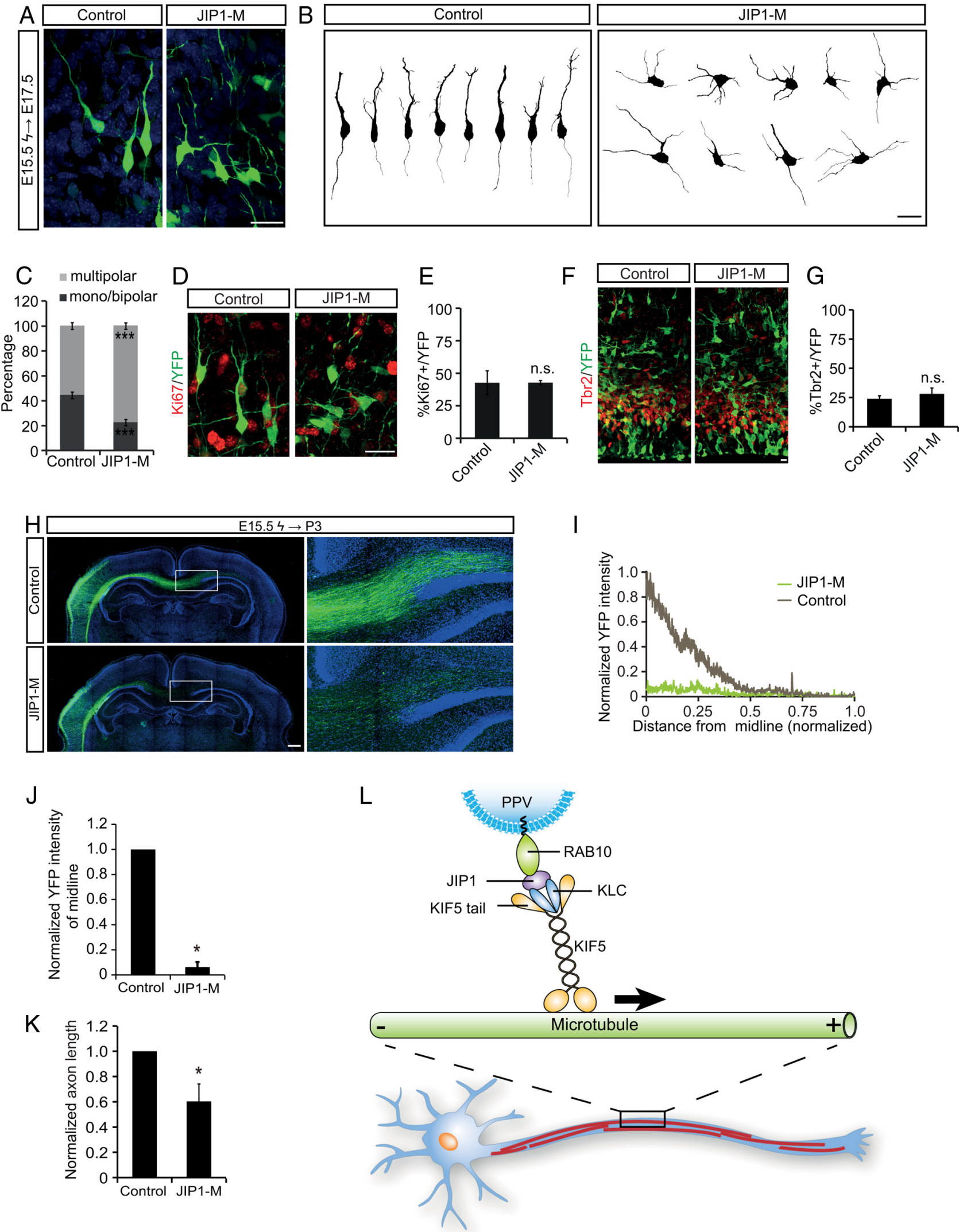

Figure 8. The KLC/JIP1/Rab10 complex is required for neuronal polarization and axon growth in vivo. $A$, Representative images of YFP-positive neurons in the IZ of E17.5 cortical sections from rat embryos, which were electroporated at E15.5 with vehicle or JIP1-M plasmids, together with PCAG-EYFP. Scale bar, $20 \mu \mathrm{m}$. $\boldsymbol{B}$, Representative tracings of YFP-positive cells in IZ regions of electroporated cortical sections. Scale bar, $20 \mu \mathrm{m}$. C, Quantification for the percentage of monopolar/bipolar and multipolar YFP-positive neurons in the SVZ and IZ of cortical sections with electroporation. Data are presented as the mean \pm SEM. ${ }^{* * *} p<0.001$, Student's $t$ test. $\boldsymbol{D}, \boldsymbol{F}$, Neocortical slices from rat embryos electroporated at E15.5 with EYFP together with indicated plasmids were stained with Ki67 antibody (D) or Tbr2 antibody (F). Scale bars: $\boldsymbol{D}, 20 \mu \mathrm{m} ; \boldsymbol{F}, 10 \mu \mathrm{m}$. $\boldsymbol{E}, \mathbf{G}$, Quantification for the percentage of Ki67-positive (Ki67+; $\boldsymbol{E}$ ) or Tbr2-positive (Tbr2 +; $\boldsymbol{G}$ ) cells among YFP-positive cells in VZ/SVZ/IZ regions of cortical sections. n.S., Not significant, Student's $t$ test. $\boldsymbol{H}$, Representative images for P3 neocortical slices from rat (Figure legend continues.) 


\section{Discussion}

It is well known that kinesin superfamily motor proteins largely govern intracellular transport of various cargos along microtubules, typically in a direction from the minus end to the plus end (Hirokawa et al., 2009). Indeed, the assembly of axonal microtubules displays a polarity, with their plus end pointing uniformly toward the growth cones of mature neurons, as opposed to the mixed polarity of dendritic microtubule polymerization (Baas, 1998). Actually, polarized microtubule assembly happens before morphological polarization (Witte et al., 2008; Lei et al., 2012). This raises a possibility that directional transport of membrane cargos following polarized microtubule assembly may determine neuronal polarization. This hypothesis is also in line with the observation that selective translocation of conventional kinesin-1 marks the initial specification of the axon (Jacobson et al., 2006). In this study, we find that directional transport of Rab10-positive vesicles is essential for polarized membrane addition during axon development.

As a scaffold protein for JNK signaling pathways, JIP1 has been shown to be a mediator for several neuronal cargos, such as JNK cascade kinases, RhoGEF190, $\beta$-amyloid precursor protein, or apolipoprotein E receptor-2 (Meyer et al., 1999; Stockinger et al., 2000; Matsuda et al., 2001; Verhey et al., 2001; Koushika, 2008). Here, we have identified Rab10 as another cargo associated with JIP1. Through direct interaction with KLC and Rab10, JIP1 couples Rab10-positive vesicles to knesin-1 motor protein, which runs along microtubules toward the distal end of nascent axon to provide the source of materials required for neuronal polarization and axon growth (Fig. $8 \mathrm{~L}$, model).

Different Rab proteins regulate various aspects of membrane trafficking events in eukaryotic cells (Grosshans et al., 2006; Stenmark, 2009). Several Rab proteins have been shown to be involved in neuronal development and functions. For example, Rab3 is known to regulate membrane trafficking and the fusion of presynaptic vesicles (Geppert et al., 1997; Schlüter et al., 2004). Rab5, Rab7, and Rab11 control endocytic pathways, which regulate neuronal morphogenesis (Shirane and Nakayama, 2006; Liu et al., 2007), migration (Kawauchi et al., 2010), or recycling of postsynaptic neurotransmitter receptors (Wang et al., 2008). We have shown recently that Rab10 is involved in polarized membrane insertion during axon development (Wang et al., 2011). JIP1 specifically interacts with Rab10 rather than other Rabs, suggesting the strictly controlled transport of individual Rab cargos.

Kinesin-1 switches from the autoinhibitory state to the active state upon loading of cargos such as JIP1 or Alcadein (Verhey et al., 2001; Araki et al., 2007). In the case of JIP1, this switch requires the cooperation of fasciculation and elongation protein $\zeta 1$, a binding partner of KIF (Blasius et al., 2007). Another layer of complexity is the phosphorylation modification of JIP1. Indeed, the JIP1-kinesin interaction has been shown to be controlled by

(Figure legend continued.) embryos electroporated at E15.5 with vehicle or JIP1-M plasmids, together with pCAG-EYFP. Enlarged areas show contralateral corpus callosum axon bundles. Scale bar, $500 \mu \mathrm{m}$. I, Intensity distribution of YFP expressed in contralateral axon bundles against the distance from the midline. The YFP intensity at the midline of the control group was normalized as 1.0.J, Quantification for the intensity of YFP in the midline. Data are presented as the mean \pm SEM. ${ }^{*} p<0.05$, Student's $t$ test. $K$, Quantification of the length of axons measured from the midline to the end of axonal tracts. The value of the control group was normalized as 1.0. Data are presented as the mean $\pm S E M .{ }^{*} p<0.05$, Student's $t$ test. $L$, Proposed model for anterograde transport of Rab10-PPVs along the axon. Rab10-PPVs are loaded to KIF5 by JIP1, which binds to KLC directly, and transported along microtubules during axon development.
JNK pathway kinases in Drosophila (Horiuchi et al., 2007). It would be of interest to determine whether Rab10 interaction with JIP1 controls kinesin-1 activity and whether JIP1 phosphorylation or other modifications regulates Rab10 loading.

Among four known JIPs, JIP3, which is structurally distinct from JIP1, also plays complex roles in axonal transport of various cargos via interaction with either KLC or KIF in Drosophila or cultured mammalian neurons (Bowman et al., 2000; Koushika, 2008; Huang et al., 2011; Sun et al., 2011). A recent report shows that, unlike JIP1, JIP3 only controls axon growth but not neuronal polarization (Sun et al., 2013). Notably, our unpublished data show that Rab10 does not interact with JIP3. Together, this study has identified Rab10 as a novel cargo carried by the JIP1 scaffold protein, which mediates anterograde transport of Rab10-PPVs, and this process is required for polarized membrane insertion during axon development.

\section{References}

Araki Y, Kawano T, Taru H, Saito Y, Wada S, Miyamoto K, Kobayashi H, Ishikawa HO, Ohsugi Y, Yamamoto T, Matsuno K, Kinjo M, Suzuki T (2007) The novel cargo Alcadein induces vesicle association of kinesin-1 motor components and activates axonal transport. EMBO J 26:14751486. CrossRef Medline

Arimura N, Kimura T, Nakamuta S, Taya S, Funahashi Y, Hattori A, Shimada A, Ménager C, Kawabata S, Fujii K, Iwamatsu A, Segal RA, Fukuda M, Kaibuchi K (2009) Anterograde transport of TrkB in axons is mediated by direct interaction with Slp1 and Rab27. Dev Cell 16:675-686. CrossRef Medline

Baas PW (1998) The role of motor proteins in establishing the microtubule arrays of axons and dendrites. J Chem Neuroanat 14:175-180. CrossRef Medline

Barnes AP, Polleux F (2009) Establishment of axon-dendrite polarity in developing neurons. Annu Rev Neurosci 32:347-381. CrossRef Medline

Blasius TL, Cai D, Jih GT, Toret CP, Verhey KJ (2007) Two binding partners cooperate to activate the molecular motor kinesin-1. J Cell Biol 176:1117. CrossRef Medline

Bowman AB, Kamal A, Ritchings BW, Philp AV, McGrail M, Gindhart JG, Goldstein LS (2000) Kinesin-dependent axonal transport is mediated by the Sunday driver (SYD) protein. Cell 103:583-594. CrossRef Medline

Bradke F, Dotti CG (1997) Neuronal polarity: vectorial cytoplasmic flow precedes axon formation. Neuron 19:1175-1186. CrossRef Medline

Cáceres A, Banker GA, Binder L (1986) Immunocytochemical localization of tubulin and microtubule-associated protein 2 during the development of hippocampal neurons in culture. J Neurosci 6:714-722. Medline

Cai D, Hoppe AD, Swanson JA, Verhey KJ (2007) Kinesin-1 structural organization and conformational changes revealed by FRET stoichiometry in live cells. J Cell Biol 176:51-63. CrossRef Medline

Chen YM, Wang QJ, Hu HS, Yu PC, Zhu J, Drewes G, Piwnica-Worms H, Luo ZG (2006) Microtubule affinity-regulating kinase 2 functions downstream of the PAR-3/PAR-6/atypical PKC complex in regulating hippocampal neuronal polarity. Proc Natl Acad Sci U S A 103:8534-8539. CrossRef Medline

Cho KI, Cai Y, Yi H, Yeh A, Aslanukov A, Ferreira PA (2007) Association of the kinesin-binding domain of RanBP2 to KIF5B and KIF5C determines mitochondria localization and function. Traffic 8:1722-1735. CrossRef Medline

Dajas-Balador F, Jones EV, Whitmarsh AJ (2008) The JIP1 scaffold protein regulates axonal development in cortical neurons. Curr Biol 18:221-226. CrossRef Medline

Dotti CG, Sullivan CA, Banker GA (1988) The establishment of polarity by hippocampal-neurons in culture. J Neurosci 8:1454-1468. Medline

Dupraz S, Grassi D, Bernis ME, Sosa L, Bisbal M, Gastaldi L, Jausoro I, Cáceres A, Pfenninger KH, Quiroga S (2009) The TC10-Exo70 complex is essential for membrane expansion and axonal specification in developing neurons. J Neurosci 29:13292-13301. CrossRef Medline

Fame RM, MacDonald JL, Macklis JD (2011) Development specification, and diversity of callosal projection neurons. Trends Neurosci 34:41-50. CrossRef Medline

Fu MM, Holzbaur EL (2013) JIP1 regulates the directionality of APP axonal 
transport by coordinating kinesin and dynein motors. J Cell Biol 202:495508. CrossRef Medline

Futerman AH, Banker GA (1996) The economics of neurite outgrowththe addition of new membrane to growing axons. Trends Neurosci 19: 144-149. CrossRef Medline

Geppert M, Goda Y, Stevens CF, Südhof TC (1997) The small GTP-binding protein Rab3A regulates a late step in synaptic vesicle fusion. Nature 387:810-814. CrossRef Medline

Glater EE, Megeath LJ, Stowers RS, Schwarz TL (2006) Axonal transport of mitochondria requires milton to recruit kinesin heavy chain and is light chain independent. J Cell Biol 173:545-557. CrossRef Medline

Grigoriev I, Splinter D, Keijzer N, Wulf PS, Demmers J, Ohtsuka T, Modesti M, Maly IV, Grosveld F, Hoogenraad CC, Akhmanova A (2007) Rab6 regulates transport and targeting of exocytotic carriers. Dev Cell 13:305314. CrossRef Medline

Grosshans BL, Ortiz D, Novick P (2006) Rabs and their effectors: achieving specificity in membrane traffic. Proc Natl Acad Sci U S A 103:1182111827. CrossRef Medline

Hirokawa N, Noda Y, Tanaka Y, Niwa S (2009) Kinesin superfamily motor proteins and intracellular transport. Nat Rev Mol Cell Biol 10:682-696. CrossRef Medline

Horiuchi D, Collins CA, Bhat P, Barkus RV, Diantonio A, Saxton WM (2007) Control of a kinesin-cargo linkage mechanism by JNK pathway kinases. Curr Biol 17:1313-1317. CrossRef Medline

Horton AC, Rácz B, Monson EE, Lin AL, Weinberg RJ, Ehlers MD (2005) Polarized secretory trafficking directs cargo for asymmetric dendrite growth and morphogenesis. Neuron 48:757-771. CrossRef Medline

Huang SH, Duan S, Sun T, Wang J, Zhao L, Geng Z, Yan J, Sun HJ, Chen ZY (2011) JIP3 mediates TrkB axonal anterograde transport and enhances BDNF signaling by directly bridging TrkB with kinesin-1. J Neurosci 31:10602-10614. CrossRef Medline

Jacobson C, Schnapp B, Banker GA (2006) A change in the selective translocation of the kinesin-1 motor domain marks the initial specification of the axon. Neuron 49:797-804. CrossRef Medline

Kanai Y, Dohmae N, Hirokawa N (2004) Kinesin transports RNA: isolation and characterization of an RNA-transporting granule. Neuron 43:513525. CrossRef Medline

Kawauchi T, Sekine K, Shikanai M, Chihama K, Tomita K, Kubo K, Nakajima K, Nabeshima Y, Hoshino M (2010) Rab GTPases-dependent endocytic pathways regulate neuronal migration and maturation through N-cadherin trafficking. Neuron 67:588-602. CrossRef Medline

Koushika SP (2008) "JIP"ing along the axon: the complex roles of JIPs in axonal transport. Bioessays 30:10-14. CrossRef Medline

Lecuit T, Pilot F (2003) Developmental control of cell morphogenesis: a focus on membrane growth. Nat Cell Biol 5:103-108. CrossRef Medline

Lei WL, Xing SG, Deng CY, Ju XC, Jiang XY, Luo ZG (2012) Laminin/beta 1 integrin signal triggers axon formation by promoting microtubule assembly and stabilization. Cell Res 22:954-972. CrossRef Medline

Liu J, Lamb D, Chou MM, Liu YJ, Li G (2007) Nerve growth factormediated neurite outgrowth via regulation of Rab5. Mol Biol Cell 18: 1375-1384. CrossRef Medline

Liu Y, Xu XH, Chen Q, Wang T, Deng CY, Song BL, Du JL, Luo ZG (2013) Myosin $\mathrm{Vb}$ controls biogenesis of post-Golgi Rab10 carriers during axon development. Nat Commun 4:2005. CrossRef Medline

Matsuda S, Yasukawa T, Homma Y, Ito Y, Niikura T, Hiraki T, Hirai S, Ohno S, Kita Y, Kawasumi M, Kouyama K, Yamamoto T, Kyriakis JM, Nishimoto I (2001) c-Jun N-terminal kinase (JNK)-interacting protein1b/islet-brain-1 scaffolds Alzheimer's amyloid precursor protein with JNK. J Neurosci 21:6597-6607. Medline

Meyer D, Liu A, Margolis B (1999) Interaction of c-Jun amino-terminal kinase interacting protein-1 with p190 rhoGEF and its localization in differentiated neurons. J Biol Chem 274:35113-35118. CrossRef Medline

Mitchell BD, Macklis JD (2005) Large-scale maintenance of dual projections by callosal and frontal cortical projection neurons in adult mice. J Comp Neurol 482:17-32. CrossRef Medline

Nadarajah B, Brunstrom JE, Grutzendler J, Wong RO, Pearlman AL (2001) Two modes of radial migration in early development of the cerebral cortex. Nat Neurosci 4:143-150. CrossRef Medline

Noctor SC, Martínez-Cerdeño V, Ivic L, Kriegstein AR (2004) Cortical neu- rons arise in symmetric and asymmetric division zones and migrate through specific phases. Nat Neurosci 7:136-144. CrossRef Medline

Pfenninger KH (2009) Plasma membrane expansion: a neuron's Herculean task. Nat Rev Neurosci 10:251-261. CrossRef Medline

Saito T, Nakatsuji N (2001) Efficient gene transfer into the embryonic mouse brain using in vivo electroporation. Dev Biol 240:237-246. CrossRef Medline

Schlüter OM, Schmitz F, Jahn R, Rosenmund C, Südhof TC (2004) A complete genetic analysis of neuronal Rab3 function. J Neurosci 24:66296637. CrossRef Medline

Setou M, Seog DH, Tanaka Y, Kanai Y, Takei Y, Kawagishi M, Hirokawa N (2002) Glutamate-receptor-interacting protein GRIP1 directly steers kinesin to dendrites. Nature 417:83-87. CrossRef Medline

Shirane M, Nakayama KI (2006) Protrudin induces neurite formation by directional membrane trafficking. Science 314:818-821. CrossRef Medline

Stenmark H (2009) Rab GTPases as coordinators of vesicle traffic. Nat Rev Mol Cell Biol 10:513-525. CrossRef Medline

Stockinger W, Brandes C, Fasching D, Hermann M, Gotthardt M, Herz J, Schneider WJ, Nimpf J (2000) The reelin receptor ApoER2 recruits JNK-interacting proteins-1 and-2. J Biol Chem 275:25625-25632. CrossRef Medline

Su Q, Cai Q, Gerwin C, Smith CL, Sheng ZH (2004) Syntabulin is a microtubule-associated protein implicated in syntaxin transport in neurons. Nat Cell Biol 6:941-953. CrossRef Medline

Sun F, Zhu C, Dixit R, Cavalli V (2011) Sunday Driver/JIP3 binds kinesin heavy chain directly and enhances its motility. EMBO J 30:3416-3429. CrossRef Medline

Sun T, Yu N, Zhai LK, Li N, Zhang C, Zhou L, Huang Z, Jiang XY, Shen Y, Chen ZY (2013) c-Jun NH2-terminal kinase (JNK)-interacting protein-3 (JIP3) regulates neuronal axon elongation in a kinesin- and JNK-dependent manner. J Biol Chem 288:14531-14543. CrossRef Medline

Taya S, Shinoda T, Tsuboi D, Asaki J, Nagai K, Hikita T, Kuroda S, Kuroda K, Shimizu M, Hirotsune S, Iwamatsu A, Kaibuchi K (2007) DISC1 regulates the transport of the NUDEL/LIS1/14-3-3 epsilon complex through kinesin-1. J Neurosci 27:15-26. CrossRef Medline

Verhey KJ, Hammond JW (2009) Traffic control: regulation of kinesin motors. Nat Rev Mol Cell Biol 10:765-777. CrossRef Medline

Verhey KJ, Lizotte DL, Abramson T, Barenboim L, Schnapp BJ, Rapoport TA (1998) Light chain-dependent regulation of Kinesin's interaction with microtubules. J Cell Biol 143:1053-1066. CrossRef Medline

Verhey KJ, Meyer D, Deehan R, Blenis J, Schnapp BJ, Rapoport TA, Margolis B (2001) Cargo of kinesin identified as JIP scaffolding proteins and associated signaling molecules. J Cell Biol 152:959-970. CrossRef Medline

Wang T, Liu Y, Xu XH, Deng CY, Wu KY, Zhu J, Fu XQ, He M, Luo ZG (2011) Lgl1 activation of rab10 promotes axonal membrane trafficking underlying neuronal polarization. Dev Cell 21:431-444. CrossRef Medline

Wang Z, Edwards JG, Riley N, Provance DW Jr, Karcher R, Li XD, Davison IG, Ikebe M, Mercer JA, Kauer JA, Ehlers MD (2008) Myosin Vb mobilizes recycling endosomes and AMPA receptors for postsynaptic plasticity. Cell 135:535-548. CrossRef Medline

Whitmarsh AJ (2006) The JIP family of MAPK scaffold proteins. Biochem Soc Trans 34:828-832. CrossRef Medline

Witte H, Neukirchen D, Bradke F (2008) Microtubule stabilization specifies initial neuronal polarization. J Cell Biol 180:619-632. CrossRef Medline

Yamada M, Toba S, Takitoh T, Yoshida Y, Mori D, Nakamura T, Iwane AH, Yanagida T, Imai H, Yu-Lee LY, Schroer T, Wynshaw-Boris A, Hirotsune S (2010) mNUDC is required for plus-end-directed transport of cytoplasmic dynein and dynactins by kinesin-1. EMBO J 29:517-531. CrossRef Medline

Ye B, Zhang YW, Jan LY, Jan YN (2006) The secretory pathway and neuron polarization. J Neurosci 26:10631-10632. CrossRef Medline

Zhang X, Zhu J, Yang GY, Wang QJ, Qian L, Chen YM, Chen F, Tao Y, Hu HS, Wang T, Luo ZG (2007) Dishevelled promotes axon differentiation by regulating atypical protein kinase C. Nat Cell Biol 9:743-754. CrossRef Medline 